\title{
On the design of discontinuous Galerkin methods for elliptic problems based on hybrid formulations
}

\author{
Ramon Codina $^{1 *}$ and Santiago Badia ${ }^{1,2}$ \\ ${ }^{1}$ Universitat Politècnica de Catalunya, Jordi Girona 1-3, Edifici C1, E-08034 Barcelona \\ ${ }^{2}$ Centre Internacional de Mètodes Numèrics en Enginyeria, Parc Mediterrani \\ de la Tecnologia, Esteve Terrades 5, E-08860 Castelldefels, Spain
}

\section{Contents}

1 Introduction $\quad 2$

2 An abstract elliptic problem in hybrid form $\quad 3$

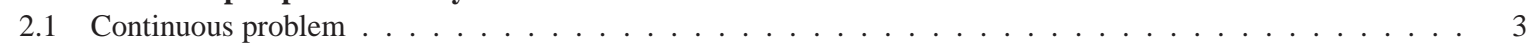

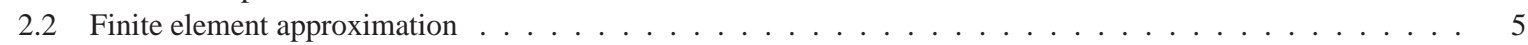

2.3 From the hybrid to the irreducible form $\ldots \ldots \ldots \ldots \ldots \ldots \ldots \ldots \ldots \ldots \ldots \ldots \ldots \ldots \ldots \ldots \ldots$

3 Scalar convection-diffusion equation (diffusion dominated case) $\quad 7$

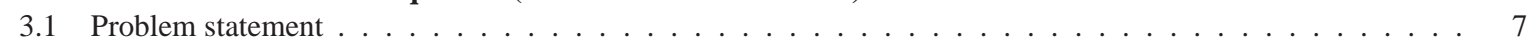

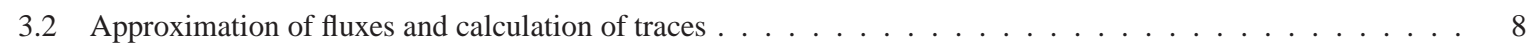

3.3 The resulting discontinuous Galerkin approximation $\ldots \ldots \ldots \ldots \ldots$

4 Stokes' problem $\quad 12$

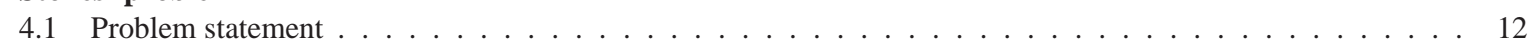

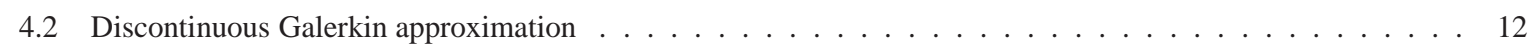

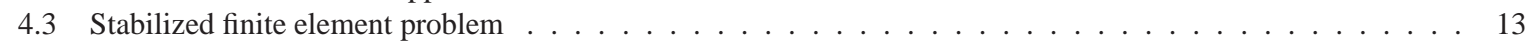

5 Darcy's problem $\quad 14$

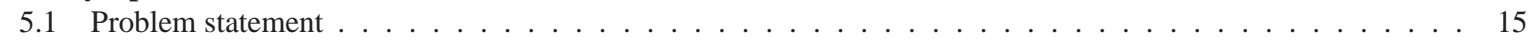

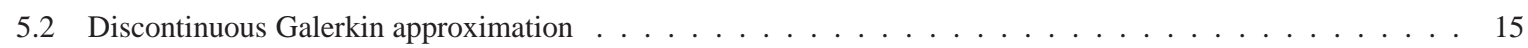

6 Maxwell's problem $\quad 16$

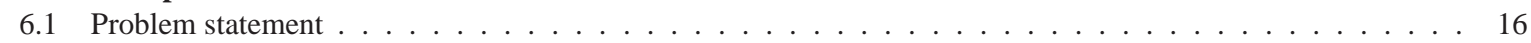

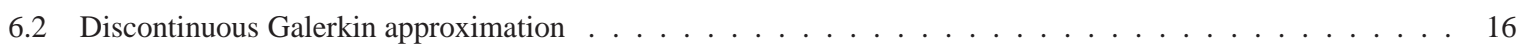

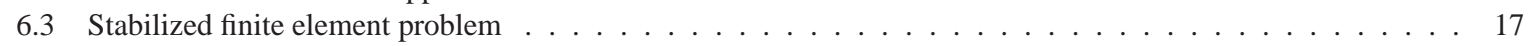

7 Numerical examples $\quad 18$

7.1 Maxwell's problem . . . . . . . . . . . . . . . . . . . . . . . . 19

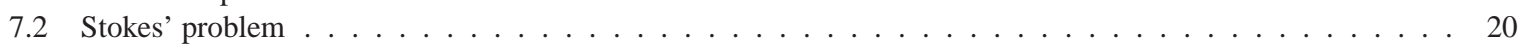

8 Conclusions $r$

\footnotetext{
${ }^{*}$ Corresponding author. E-mail: ramon.codina@upc.edu, Tel: +34 934016486
} 


\begin{abstract}
The objective of this paper is to present a framework for the design of discontinuous Galerkin (dG) methods for elliptic problems. The idea is to start from a hybrid formulation of the problem involving as unknowns the main field in the interior of the element domains and its fluxes and traces on the element boundaries. Rather than working with this three-field formulation, fluxes are modeled using finite difference expressions and then the traces are determined by imposing continuity of fluxes, although other strategies could be devised. This procedure is applied to four elliptic problems, namely, the convection-diffusion equation (in the diffusion dominated regime), the Stokes problem, the Darcy problem and the Maxwell problem. We justify some well known $\mathrm{dG}$ methods with some modifications that in fact allow to improve the performance of the original methods, particularly when the physical properties are discontinuous.
\end{abstract}

Keywords: Discontinuous Galerkin, elliptic problems, hybrid formulations, stabilized finite element methods

\title{
1 Introduction
}

The aim of this work is to present a methodology for designing discontinuous Galerkin (dG) methods for elliptic problems. The starting point is a three-field hybrid formulation of the problem, in which the unknowns are the main field in the interior of the element domains of the finite element partition, their fluxes on the interelement edges (faces, in 3D) and their traces also on these edges.

Instead of considering the fluxes and the traces as independent variables, they are related to the main field in the interior of the element domains, which may be interpolated in a discontinuous manner. The way to design this relationship is as follows. First, fluxes are written in terms of the main variable and its traces. The interesting point is when derivatives are needed to compute these fluxes. It is required to reconstruct the unknown from the discontinuous approximation and the traces. A particular way to do this is proposed here, which consists of assuming that the discontinuous approximation is valid up to a certain distance to the element boundary, from where the traces are used for the reconstruction. The derivatives of the reconstructed variable can now be approximated using finite difference expressions, this leading to an expression of the fluxes in terms of the discontinuous approximation and the traces.

Once the fluxes are given, the traces can be obtained by imposing continuity of fluxes. The expressions for the fluxes and traces in terms of the discontinuous approximation can be also extended to their test functions, which can be expressed in terms of the test functions of the discontinuous approximation. When these expressions are inserted into the hybrid discrete problem, a problem posed in terms of the main field in the interior of the element domains is obtained. We show that several $\mathrm{dG}$ methods can be obtained following this procedure (see [1]), with some modifications that in fact improve their performance and which are commented later. The methodology described is then applied to four elliptic problems, namely, the convection-diffusion equation (in the diffusion dominated regime), the Stokes problem, the Darcy problem and the Maxwell problem.

Apart from the design of dG formulations, the present framework can also be used for the design of stabilized finite element method including stabilization terms defined on the element boundaries [16] or for the formulation of new transmission conditions in domain interaction problems (with applications for example to domain decomposition and fluid-structure interaction) [15].

The organization of the paper is as follows. In Section 2 we describe the hybrid formulation of an abstract elliptic problem that serves as starting point for the methodology we propose. We also describe our "program" in this section. The ideas outlined are then applied to four elliptic problems, namely, the convection-diffusion equation in the diffusion dominated regime (Section 3), the Stokes problem (Section 4), the Darcy problem (Section 5) and the Maxwell problem (Section 6). Some simple numerical examples are presented in Section 7, which are intended to show the improvement in the 
behavior of domain decomposition preconditioners for problems with physical coefficient jumps when a modification motivated by the present approach is used. Conclusions close the paper in Section 8.

\section{An abstract elliptic problem in hybrid form}

\subsection{Continuous problem}

The numerical approximation we propose can be motivated from a hybrid formulation of the problem (see $[8,9]$ ). Let us now introduce it at the continuous level, prior to any numerical approximation. Assume that we have a boundary value problem posed in a domain $\Omega \subset \mathbb{R}^{d}(d=2,3)$, which we will assume polyhedral in the finite element approximation, split as $\bar{\Omega}=\bar{\Omega}_{1} \cup \bar{\Omega}_{2}$, and with $\Gamma=\partial \Omega_{1} \cap \partial \Omega_{2}$ (see Fig. 1).

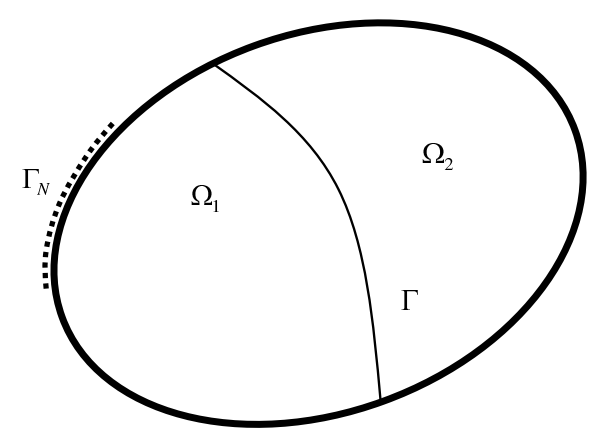

Figure 1: Splitting of the domain

Let $X$ be a space of functions defined on $\Omega$, which in our applications will be a classical Sobolev space. The notation $(\cdot, \cdot)_{\omega}$ and $\langle\cdot, \cdot\rangle_{\omega}$ will respectively denote the $L^{2}(\omega)$ inner product and the integral in a region $\omega$ of the product of two functions, this case including the duality pairing. The subscript $\omega$ will be deleted when $\omega=\Omega$. The norm in the functional space $X$ will be indicated by $\|\cdot\|_{X}$.

Consider an abstract variational problem consisting in finding an unknown $u$ in $X$ such that

$$
B(u, v)=L(v) \quad \forall v \in X,
$$

where $B(u, v)$ is a bilinear form on $X \times X$ and $L$ a linear form defined on $X$, both assumed to be continuous. If the problem considered involves Dirichlet boundary conditions, we will assume that they are homogeneous and enforced in an essential manner, that is to say, included in the definition of $X$. However, at the numerical level it would be straightforward to extend the method to be introduced to a penalty method to impose such conditions.

Let $u_{i}, v_{i}$ be the restrictions of $u, v \in X$ to subdomain $\Omega_{i}$, and $X_{i}$ the spaces where they belong, $i=1,2$. Suppose that $u \in X$ has a well defined trace on $\Gamma$ belonging to a space $T$, and a flux corresponding to the differential operator associated to (1) belonging to a space $F_{i}$ when computed from subdomain $\Omega_{i}, i=1,2$. Then, the hybrid formulation of (1) that we consider is the following: find 
$u_{i} \in X_{i}, \lambda_{i} \in F_{i}, i=1,2$, and $\gamma \in T$ such that

$$
\begin{array}{ll}
B_{1}\left(u_{1}, v_{1}\right)-\left\langle\lambda_{1}, v_{1}\right\rangle_{\Gamma}=l_{1}\left(v_{1}\right) & \forall v_{1} \in X_{1}, \\
B_{2}\left(u_{2}, v_{2}\right)-\left\langle\lambda_{2}, v_{2}\right\rangle_{\Gamma}=l_{2}\left(v_{2}\right) & \forall v_{2} \in X_{2}, \\
\left\langle\mu_{1}, u_{1}-\gamma\right\rangle_{\Gamma}=0 & \forall \mu_{1} \in F_{1}, \\
\left\langle\mu_{2}, u_{2}-\gamma\right\rangle_{\Gamma}=0 & \forall \mu_{2} \in F_{2}, \\
\left\langle\kappa, \lambda_{1}+\lambda_{2}\right\rangle_{\Gamma}=0 & \forall \kappa \in T,
\end{array}
$$

where $B_{i}$ and $L_{i}$ are the restrictions of $B$ and $L$ to $X_{i} \times X_{i}$ and $X_{i}$, respectively.

If the problem includes imposition of fluxes of $u$ in a part $\Gamma_{N}$ of $\partial \Omega$, which for the sake of simplicity we may consider contained in $\partial \Omega_{1}$ (see Fig. 1), this imposition may be also "hybridized", yielding the problem

$$
\begin{array}{ll}
B_{1}\left(u_{1}, v_{1}\right)-\left\langle\lambda_{1}, v_{1}\right\rangle_{\Gamma}-\left\langle\lambda_{N}, v_{1}\right\rangle_{\Gamma_{N}}=L_{1}\left(v_{1}\right) & \forall v_{1} \in X_{1}, \\
B_{2}\left(u_{2}, v_{2}\right)-\left\langle\lambda_{2}, v_{2}\right\rangle_{\Gamma}=L_{2}\left(v_{2}\right) & \forall v_{2} \in X_{2}, \\
\left\langle\mu_{1}, u_{1}-\gamma\right\rangle_{\Gamma}+\left\langle\mu_{1}, u_{1}-\gamma\right\rangle_{\Gamma_{N}}=0 & \forall \mu_{1} \in F_{1}, \\
\left\langle\mu_{2}, u_{2}-\gamma\right\rangle_{\Gamma}=0 & \forall \mu_{2} \in F_{2}, \\
\left\langle\kappa, \lambda_{1}+\lambda_{2}\right\rangle_{\Gamma}+\left\langle\kappa, \lambda_{N}\right\rangle_{\Gamma_{N}}=\langle\kappa, q\rangle_{\Gamma_{N}} & \forall \kappa \in T,
\end{array}
$$

where $q$ is the flux to be prescribed. In this case, the linear form $L$ (and the forms $L_{1}$ and $L_{2}$ resulting from the splitting of the domain) does not include the prescription of the fluxes, and $T$ includes functions defined on $\Gamma_{N}$.

Concerning the well posedness of the problem (for simplicity with $\Gamma_{N}=\emptyset$ ), let us write it as a single variational equation of the form

$$
B_{\text {hyb }}\left(u_{1}, u_{2}, \lambda_{1}, \lambda_{2}, \gamma ; v_{1}, v_{2}, \mu_{1}, \mu_{2}, \kappa\right)=L_{\text {hyb }}\left(v_{1}, v_{2}, \mu_{1}, \mu_{2}, \kappa\right),
$$

where

$$
\begin{aligned}
B_{\mathrm{hyb}}\left(u_{1}, u_{2}, \lambda_{1}, \lambda_{2}, \gamma ; v_{1}, v_{2}, \mu_{1}, \mu_{2}, \kappa\right) & :=B_{1}\left(u_{1}, v_{1}\right)-\left\langle\lambda_{1}, v_{1}\right\rangle_{\Gamma}+B_{2}\left(u_{2}, v_{2}\right)-\left\langle\lambda_{2}, v_{2}\right\rangle_{\Gamma} \\
& +\left\langle\mu_{1}, u_{1}-\gamma\right\rangle_{\Gamma}+\left\langle\mu_{2}, u_{2}-\gamma\right\rangle_{\Gamma}+\left\langle\kappa, \lambda_{1}+\lambda_{2}\right\rangle_{\Gamma}, \\
L_{\mathrm{hyb}}\left(v_{1}, v_{2}, \mu_{1}, \mu_{2}, \kappa\right) & :=L_{1}\left(v_{1}\right)+L_{2}\left(v_{2}\right),
\end{aligned}
$$

with $\left(u_{1}, u_{2}, \lambda_{1}, \lambda_{2}, \gamma\right) \in X_{1} \times X_{2} \times F_{1} \times F_{2} \times T$ and which must hold for all $\left(v_{1}, v_{2}, \mu_{1}, \mu_{2}, \kappa\right) \in$ $X_{1} \times X_{2} \times F_{1} \times F_{2} \times T$. If we assume that $B_{i}\left(u_{i}, v_{i}\right)$ are coercive in $X_{i} \times X_{i}(i=1,2)$, we have that

$$
B_{\text {hyb }}\left(u_{1}, u_{2}, \lambda_{1}, \lambda_{2}, \gamma ; u_{1}, u_{2}, \lambda_{1}, \lambda_{2}, \gamma\right)=B_{1}\left(u_{1}, u_{1}\right)+B_{2}\left(u_{2}, u_{2}\right) \gtrsim\left\|u_{1}\right\|_{X_{1}}^{2}+\left\|u_{2}\right\|_{X_{2}}^{2},
$$

where $\gtrsim$ is used to denote $\geq$ up to positive constants, and $\lesssim$ will be used analogously. Control on $\left\|\lambda_{1}\right\|_{F_{1}},\left\|\lambda_{2}\right\|_{F_{2}}$ and $\|\gamma\|_{T}$ can be obtained only if the inf-sup conditions

$$
\begin{aligned}
& \inf _{\mu_{i} \in F_{i} \backslash\{0\}} \sup _{v_{i} \in V_{i} \backslash\{0\}} \frac{\left\langle\mu_{i}, v_{i}\right\rangle_{\Gamma}}{\left\|\mu_{i}\right\|_{F_{i}}\left\|v_{i}\right\|_{X_{i}}} \geq C_{F}, \quad i=1,2, \\
& \inf _{\kappa \in T \backslash\{0\}} \sup _{\mu_{i} \in F_{i}, \mu_{1}+\mu_{2} \neq 0} \frac{\left\langle\kappa, \mu_{1}+\mu_{2}\right\rangle_{\Gamma}}{\|\kappa\|_{T}\left(\left\|\mu_{1}\right\|_{F_{1}}+\left\|\mu_{2}\right\|_{F_{2}}\right)} \geq C_{T},
\end{aligned}
$$

hold for positive constants $C_{F}$ and $C_{T}$. Under assumptions (8) and (9), the coercivity of $B_{i}\left(u_{i}, v_{i}\right)$ in $X_{i} \times X_{i}(i=1,2)$ and appropriate continuity of all the forms involved in the problem, a well posedness result for (7) can be easily proved. When $B_{i}\left(u_{i}, v_{i}\right)$ are not coercive, but the problem corresponds to a well posed saddle point problem, this well posedness can also be established. 


\subsection{Finite element approximation}

Let $\mathcal{P}_{h}=\{K\}$ be a shape regular finite element partition of the domain $\Omega$ of diameter $h$. Let also $\mathcal{E}_{h}=\{E\}$ be the collection of edges associated to $\mathcal{P}_{h}$. In particular, we will be interested in edges interior to $\Omega$, which will be labelled $E_{0}$, and edges on $\Gamma_{N}$, which will be labelled $E_{N}$. Summation over all the elements will be indicated as $\sum_{K}$ and summation over the edges as $\sum_{E}$. Consistently with the notation introduced previously, the symbol $\left\langle f_{1}, f_{2}\right\rangle_{D}$ will be used to denote the integral of the product of functions $f_{1}$ and $f_{2}$ over $D$, with $D=K$ (an element), $D=\partial K$ (an element boundary) or $D=E$ (an edge).

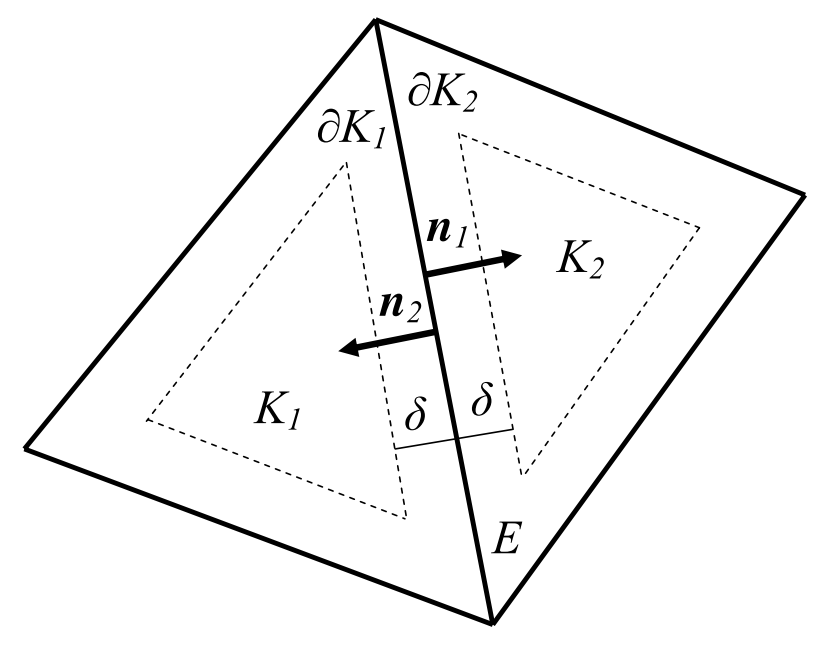

Figure 2: Notation for the approximation of fluxes and traces for interior edges

Suppose now that elements $K_{1}$ and $K_{2}$ share an edge $E$, and let $\boldsymbol{n}_{1}$ and $\boldsymbol{n}_{2}$ be the normals to $E$ exterior to $K_{1}$ and $K_{2}$, respectively (see Fig. 2). For a scalar function $f$, possibly discontinuous across $E$, we define its jump as $\llbracket \boldsymbol{n} f \rrbracket_{E}:=\left.\boldsymbol{n}_{1} f\right|_{\partial K_{1} \cap E}+\left.\boldsymbol{n}_{2} f\right|_{\partial K_{2} \cap E}$, and for a vector or tensor $\boldsymbol{v}$, $\llbracket \boldsymbol{n} \cdot \boldsymbol{v} \rrbracket_{E}:=\left.\boldsymbol{n}_{1} \cdot \boldsymbol{v}\right|_{\partial K_{1} \cap E}+\left.\boldsymbol{n}_{2} \cdot \boldsymbol{v}\right|_{\partial K_{2} \cap E}$. Averages of functions discontinuous across an edge $E$ will be denoted by $\{f\}_{E}=\frac{1}{2}\left(\left.f\right|_{\partial K_{1} \cap E}+\left.f\right|_{\partial K_{2} \cap E}\right)$, omitting the subscript $E$ when there is no possibility of confusion. Note that we could write $\llbracket \boldsymbol{n} f \rrbracket_{E}=2\{\boldsymbol{n} f\}_{E}$, although we will keep the notation $\llbracket \boldsymbol{n} f \rrbracket_{E}$ to stress the presence of the normal $\boldsymbol{n}$, which gives the meaning of jump to this expression. Note also that it is common to omit the normal in the definition of $\llbracket n f \rrbracket_{E}$, so that the jump of a scalar function is a vector [1].

We can easily extend formulation (2)-(6) to multiple subdomains and, in particular, to the case in which each of these is an element domain of $\mathcal{P}_{h}$. The resulting hybrid formulation can then be approximated using a finite element method. Let $V_{h}, F_{h}$ and $T_{h}$ be the spaces where $u_{h}, \lambda_{h}$ and $\gamma_{h}$ are sought, respectively. Note that $F_{h}$ is made of functions that may be multi-valuate on the edges of the finite element partition, whereas $T_{h}$ is made of functions uniquely defined on these edges. These spaces will be endowed with the appropriate norm depending on the problem considered.

The extension of the hybrid formulation to the elements of the finite element partition reads as 
follows: find $\left(u_{h}, \lambda_{h}, \gamma_{h}\right) \in V_{h} \times F_{h} \times T_{h}$ such that

$$
\begin{aligned}
& \sum_{K} B_{K}\left(u_{h}, v_{h}\right)-\sum_{K}\left\langle\lambda_{h}, v_{h}\right\rangle_{\partial K}=\sum_{K} L_{K}\left(v_{h}\right), \\
& \sum_{K}\left\langle\kappa_{h}, \lambda_{h}\right\rangle_{\partial K}=\left\langle\kappa_{h}, q\right\rangle_{\Gamma_{N}}, \\
& \sum_{K}\left\langle\mu_{h}, u_{h}-\gamma_{h}\right\rangle_{\partial K}=0
\end{aligned}
$$

for all $\left(v_{h}, \mu_{h}, \kappa_{h}\right) \in V_{h} \times F_{h} \times T_{h}$.

\subsection{From the hybrid to the irreducible form}

The objective of this work can be summarized by saying that we intend to design dG methods based on the hybrid finite element approximation (10)-(12). To this end, the key point to step to the method we propose is to design expressions to compute the fluxes $\lambda_{h}$ and the traces $\gamma_{h}$ from finite difference-like approximations. The steps we propose are:

1. Compute the fluxes $\lambda_{h}$ from values of $u_{h}, \gamma_{h}$ using finite difference-like expressions.

2. Compute the traces $\gamma_{h}$ by imposing continuity of total fluxes, including Neumann boundary conditions where they are applied.

3. Go to the expression obtained in 1 with the expression of $\gamma_{h}$ just obtained to compute $\lambda_{h}$ in terms of $u_{h}$.

This general procedure will be applied in the following sections to different elliptic problems. Before proceeding, let us remark that step 2 will allow us to significantly simplify the problem. Suppose that $\lambda_{h}=\Lambda_{h}\left(u_{h}\right)$ is the expression of the fluxes in terms of $u_{h}$ obtained, for simplicity without Neumann boundary conditions. If we can satisfy exactly the continuity of these fluxes and take the flux test function $\mu_{h}=\Lambda_{h}\left(v_{h}\right), v_{h}$ being the test function for $u_{h}$, then (11) will be exactly satisfied and $\sum_{K}\left\langle\mu_{h}, \gamma_{h}\right\rangle_{\partial K}=0$. The problem reduces then to find $u_{h} \in V_{h}$ such that

$$
\sum_{K} B_{K}\left(u_{h}, v_{h}\right)-\sum_{K}\left\langle\Lambda_{h}\left(u_{h}\right), v_{h}\right\rangle_{\partial K}-\sum_{K}\left\langle\Lambda_{h}\left(v_{h}\right), u_{h}\right\rangle_{\partial K}=\sum_{K} L_{K}\left(v_{h}\right),
$$

for all $v_{h} \in V_{h}$, where we have added up (10) and what remains of (12) because $\mu_{h}$ is not independent of $v_{h}$. The sign of the third term in the left-hand-side of this expression has been chosen to get a symmetric problem when $B_{K}$ is symmetric.

A possible way to satisfy the exact continuity of fluxes is to take them of the form $\lambda_{h}=\Lambda_{h}\left(u_{h}\right)=$ $\boldsymbol{n} \cdot \boldsymbol{\Lambda}_{h}^{0}\left(u_{h}\right)$, with $\boldsymbol{\Lambda}_{h}^{0}\left(u_{h}\right)$ a single-valued vector field defined on the element edges. This will be precisely the form of the fluxes we will propose.

When there are Neumann boundary conditions, a particular expression for the fluxes $\lambda_{h}$ will be obtained, the corresponding test function being zero. This results in the classical boundary term corresponding to the prescription of fluxes that needs to be added to (13). This equation will be the starting point of our developments.

Remark 1 Most dG methods are motivated by integration-by-parts of the differential equation tested with a test function $v_{h}$ within each element, which leads to the first term in the left-hand-side of (13) and, as we shall see, part of the second. Then (part of) the third term is introduced to ensure symmetry, provided $\llbracket \boldsymbol{n} u \rrbracket_{E}=0$ for the solution of the continuous problem $u$. After this, additional "stability" 
terms need to be added to ensure appropriate continuity of the unknown (in a weak sense) [7]. In our case, these terms will appear naturally in the definition of $\Lambda_{h}\left(u_{h}\right)$, whose design will be "non-classical" in order to satisfy (11) exactly. Moreover, even though it does not appear in (13), we will have at our disposal a definition of a trace.

Remark 2 Other hybrid formulations can be used as starting point to design dG methods. In particular, in [12] a hybrid formulation is proposed for the diffusion equation, which is extended in $[11,23]$ to the convection-diffusion equation. The hybridization of the problem is completely different to our threefield approach, introduced first in [16] for the same problem. Our unknowns are the field in the interior of the elements and its traces and fluxes on the boundaries, whereas in the above references it is the unknown and the fluxes in the interior (dual mixed formulation) and the traces on the boundaries. Note that our objective is to motivate standard dG methods with some modifications, whereas a non-standard formulation is proposed in $[12,11,23]$.

\section{Scalar convection-diffusion equation (diffusion dominated case)}

In this section we apply the previous idea to the convection-diffusion equation in the diffusion dominated regime, the simplest elliptic problem. Convection and reaction and only introduced for generality, without considering the instabilities that may arise when either of these terms dominates the diffusive one.

\subsection{Problem statement}

The boundary value problem we consider is: find $u: \Omega \longrightarrow \mathbb{R}$ such that

$$
\begin{array}{ll}
\mathcal{L}(u):=-\nabla \cdot(k \nabla u)+\boldsymbol{a} \cdot \nabla u+s u=f & \text { in } \Omega, \\
u=0 & \text { on } \Gamma_{D}, \\
\mathcal{T}(u):=k \partial_{n} u=q & \text { on } \Gamma_{N},
\end{array}
$$

where $k>0$ is the diffusion coefficient, $\boldsymbol{a} \in \mathbb{R}^{d}$ the advection velocity and $s \geq 0$ the reaction (absorption) coefficient. All these coefficients may be considered piecewise constant within each element of the finite element partition, for simplicity. The boundary is split as $\partial \Omega=\Gamma_{D} \cup \Gamma_{N}$, and $\partial_{n} \equiv \boldsymbol{n} \cdot \nabla$.

The irreducible weak form of the problem reads: find $u \in V=\left\{v \in H^{1}(\Omega)|v|_{\Gamma_{D}}=0\right\}$ such that

$$
B(u, v)=L(v) \quad \forall v \in V,
$$

where

$$
\begin{aligned}
B(u, v) & :=(k \nabla u, \nabla v)+(\boldsymbol{a} \cdot \nabla u, v)+(s u, v), \\
L(v) & :=\langle f, v\rangle+\langle q, v\rangle_{\Gamma_{N}} .
\end{aligned}
$$

The hybrid formulation corresponding to (2)-(6) in this case is posed in the spaces $V$ defined above, $T=H_{00}^{1 / 2}(\Gamma)$ and $F_{i}=\left(H_{00}^{1 / 2}\left(\Gamma_{i}\right)\right)^{\prime}$ (the prime denoting dual space), with $\Gamma_{i}=\partial \Omega_{i} \cap \Omega$. The solution of the hybrid problem is $\gamma=\left.u_{1}\right|_{\Gamma_{1}}=\left.u_{2}\right|_{\Gamma_{2}}, \lambda_{1}=-\lambda_{2}=\left.k_{1} \boldsymbol{n}_{1} \cdot \nabla u_{1}\right|_{\Gamma_{1}}=-\left.k_{2} \boldsymbol{n}_{2} \cdot \nabla u_{2}\right|_{\Gamma_{2}}$. The extension to a hybrid problem considering all subdomains of the finite element partition (problem (10)(12)) is also immediately set. Abusing of the notation, we can consider this same problem as a finite 
element approximation of the hybrid continuous one, the finite element spaces being

$$
\begin{aligned}
& V_{h}=\left\{v_{h}: \bigcup K \longrightarrow \mathbb{R}\left|v_{h}\right|_{K} \in P_{p_{d}}(K), K \in \mathcal{P}_{h}\right\}, \\
& F_{h}=\left\{\mu_{h}: \bigcup \partial K \longrightarrow \mathbb{R}\left|\mu_{h}\right|_{\partial K} \in P_{p_{f}}(\partial K), K \in \mathcal{P}_{h}\right\}, \\
& T_{h}=\left\{\kappa_{h}: \bigcup E \longrightarrow \mathbb{R}\left|\kappa_{h}\right|_{E} \in P_{p_{t}}(E), E \in \mathcal{E}_{h}, \text { and }\left.\kappa_{h}\right|_{E}=0 \text { if } E \subset \Gamma_{D}\right\},
\end{aligned}
$$

where $P_{p}(\omega)$ is the set of polynomials of degree $p$ on the domain $\omega$.

\subsection{Approximation of fluxes and calculation of traces}

Let us apply the methodology described in Subsection 2.3 to the convection-diffusion equation. Assume first that we have an interior edge, the situation depicted in Fig. 2. Suppose that this edge $E$ is shared by two elements $K_{1}, K_{2} \in \mathcal{P}_{h}$. Let us take now a normal direction across edge $E$, either $\boldsymbol{n}_{1}$ or $\boldsymbol{n}_{2}$. Values of the unknowns and the coefficient $k$ will be denoted with the superscript - or + depending on whether they belong to the left or right side of $E$ with respect to the normal chosen.

We assume that the approximation $u_{h}$ is meaningful to compute the fluxes $\lambda_{h}$ up to a distance $\delta$ to edge E, on which the trace takes a value $\gamma_{h}$. The distance $\delta$ will be a parameter of the formulation. Using an approximation of order $\mathcal{O}(\delta)$, the fluxes can be approximated as

$$
\lambda_{h}^{ \pm} \approx \frac{k^{ \pm}}{\delta}\left(\gamma_{h}-u_{h, \delta}^{ \pm}\right),
$$

where $u_{h, \delta}^{+}$and $u_{h, \delta}^{-}$are the values of $u_{h}$ at a distance $\delta$ from $E$. These can be computed from a Taylor expansion as (see Figure 3):

$$
u_{h, \delta}^{ \pm}=u_{h}^{ \pm}-\delta \partial_{n^{ \pm}} u_{h}^{ \pm}+\mathcal{O}\left(\delta^{2}\right),
$$

where $\partial_{n^{+}}$is the derivative in the direction of the normal chosen and $\partial_{n^{-}}$the opposite. Therefore:

$$
\lambda_{h}^{ \pm} \approx \frac{k^{ \pm}}{\delta}\left(\gamma_{h}-u_{h}^{ \pm}\right)+k^{ \pm} \partial_{n^{ \pm}} u_{h}^{ \pm} .
$$

Equations (15) complete the first step of the strategy described earlier. It is obvious that other possibilities could be used. In particular, if $u_{h, 2 \delta}$ is the value of $u_{h}$ at a distance $2 \delta$ from the edge, we could approximate $\lambda_{h}^{ \pm}=\frac{k}{2 \delta}\left(3 \gamma_{h}-4 u_{h, \delta}^{ \pm}+u_{h, 2 \delta}^{ \pm}\right)+\mathcal{O}\left(\delta^{2}\right)$. Using the expansions $u_{h, \delta}^{ \pm}=$ $u_{h}^{ \pm}-\delta \partial_{n^{ \pm}} u_{h}^{ \pm}+\frac{1}{2} \delta^{2} \partial_{n^{ \pm}}^{2} u_{h}^{ \pm}+\mathcal{O}\left(\delta^{3}\right)$ and $u_{h, 2 \delta}^{ \pm}=u_{h}^{ \pm}-2 \delta \partial_{n^{ \pm}} u_{h}^{ \pm}+2 \delta^{2} \partial_{n^{ \pm}}^{2} u_{h}^{ \pm}+\mathcal{O}\left(\delta^{3}\right)$, we would obtain $\lambda_{h}^{ \pm}=\frac{3 k^{ \pm}}{2 \delta}\left(\gamma_{h}-u_{h}^{ \pm}\right)+k^{ \pm} \partial_{n^{ \pm}} u_{h}^{ \pm}+\mathcal{O}\left(\delta^{2}\right)$ instead of (15). The change is only in the factor that multiplies $1 / \delta$, which is a numerical parameter. Thus, we may keep (15) for our purposes.

The second step consists of imposing continuity of total fluxes. For the continuous problem, this amounts to say that $\llbracket \boldsymbol{n} \cdot k \nabla u \rrbracket \equiv \llbracket \mathcal{T}(u) \rrbracket=0$. Note that here we use the symbol $\llbracket \cdot \rrbracket$ understanding that $\mathcal{T}(u)$ contains the normal in its definition. Using expressions (15) and going back to the notation in Fig. 2, the approximate continuity of fluxes leads to

$$
\begin{aligned}
0 & =\llbracket \mathcal{T}(u) \rrbracket \\
& \approx \lambda_{h, 1}+\lambda_{h, 2} \\
& \approx \frac{1}{\delta}\left[\left(k_{1}+k_{2}\right) \gamma_{h}-k_{1} u_{h, 1}-k_{2} u_{h, 2}\right]+\llbracket \mathcal{T}\left(u_{h}\right) \rrbracket \\
& =\llbracket \mathcal{T}\left(u_{h}\right) \rrbracket+\frac{2}{\delta}\left(\{k\} \gamma_{h}-\left\{k u_{h}\right\}\right) .
\end{aligned}
$$




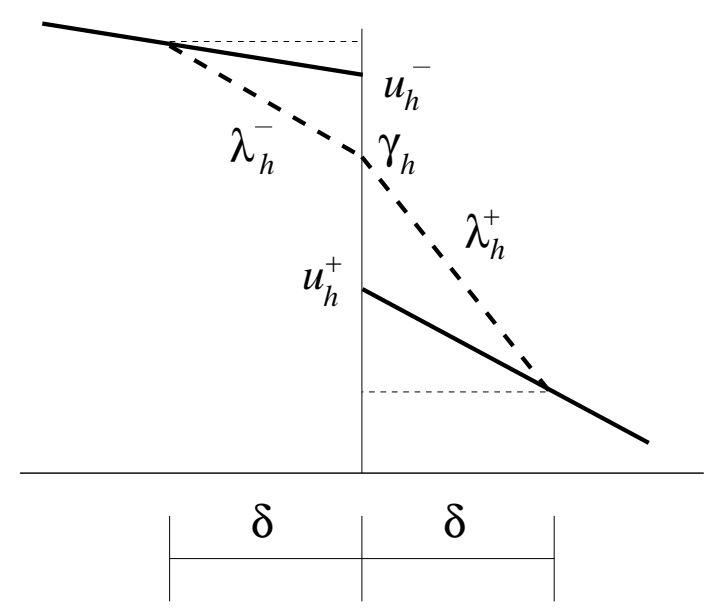

Figure 3: Approximation of fluxes

This allows us to obtain the approximation of the traces on each edge we were looking for:

$$
\gamma_{h}=\frac{\left\{k u_{h}\right\}}{\{k\}}-\frac{\delta}{2\{k\}} \llbracket \mathcal{T}\left(u_{h}\right) \rrbracket .
$$

Here and below, we will use the symbol = for our models to compute fluxes and traces, understanding that they are approximations to the exact expressions.

Inserting (17) into (15) we see that the fluxes on the boundaries of the elements can be computed as

$$
\begin{aligned}
\lambda_{h} & =\frac{k}{\delta}\left(\gamma_{h}-u_{h}\right)+\mathcal{T}\left(u_{h}\right) \\
& =\frac{k}{\delta}\left(\frac{\left\{k u_{h}\right\}}{\{k\}}-u_{h}\right)-\frac{k}{2\{k\}} \llbracket \mathcal{T}\left(u_{h}\right) \rrbracket+\mathcal{T}\left(u_{h}\right) .
\end{aligned}
$$

This expression can be written in a more suggestive way. Consider the harmonic average:

$$
\langle k\rangle:=\frac{k_{1} k_{2}}{\{k\}}=\frac{2 k_{1} k_{2}}{k_{1}+k_{2}} .
$$

We may write, after some algebraic manipulations (considering for example element $K_{1}$ ):

$$
\begin{aligned}
\frac{k_{1}}{\delta}\left(\frac{\left\{k u_{h}\right\}}{\{k\}}-u_{h, 1}\right) & =-\frac{\langle k\rangle}{2 \delta} \boldsymbol{n}_{1} \cdot \llbracket \boldsymbol{n} u_{h} \rrbracket, \\
-\frac{k_{1}}{2\{k\}} \llbracket \mathcal{T}\left(u_{h}\right) \rrbracket+\mathcal{T}\left(u_{h}\right)_{1} & =\langle k\rangle \boldsymbol{n}_{1} \cdot\left\{\nabla u_{h}\right\} .
\end{aligned}
$$

We finally obtain:

$$
\lambda_{h}=-\frac{\langle k\rangle}{2 \delta} \boldsymbol{n} \cdot \llbracket \boldsymbol{n} u_{h} \rrbracket+\langle k\rangle \boldsymbol{n} \cdot\left\{\nabla u_{h}\right\} \cdot
$$

Equations (17) and (18) are the expressions sought in the case of interior edges. Note that (18) is in fact defined on the boundaries of the elements, and therefore the value on the edge is not uniquely defined. In fact, as it was mentioned earlier, these fluxes are of the form $\lambda_{h}=\boldsymbol{n} \cdot \boldsymbol{\Lambda}_{h}^{0}\left(u_{h}\right)$, with $\boldsymbol{\Lambda}_{h}^{0}\left(u_{h}\right)=-\frac{\langle k\rangle}{2 \delta} \llbracket \boldsymbol{n} u_{h} \rrbracket+\langle k\rangle\left\{\nabla u_{h}\right\}$ single-valued. 
Suppose now that the edge we consider is on the Neumann boundary, that is to say, $E \subset \Gamma_{N}$. The continuity of total fluxes has to be replaced by the equilibrium of fluxes in the case of Neumann edges. Proceeding as above, we now have that

$$
\lambda_{h}=q .
$$

This expression is defined for the part of the element boundaries that belongs to $\Gamma_{N}$. From the approximation of $\lambda_{h}$, which now does not depend on the traces, we have:

$$
q=\lambda_{h} \approx \mathcal{T}\left(u_{h}\right)+\frac{k}{\delta}\left(\gamma_{h}-u_{h}\right) .
$$

Therefore:

$$
\gamma_{h}=u_{h}-\frac{\delta}{k}\left(\mathcal{T}\left(u_{h}\right)-q\right)
$$

\subsection{The resulting discontinuous Galerkin approximation}

The original hybrid formulation can be written in a compact form as indicated in (13) with $\Lambda\left(v_{h}\right)$ given by (18) or (19).

Let us collect the expressions obtained. The fluxes and traces for interior edges are given by:

$$
\begin{aligned}
& \gamma_{h}=\frac{\left\{k u_{h}\right\}}{\{k\}}-\frac{\delta}{2\{k\}}\left(\llbracket k \boldsymbol{n} \cdot \nabla u_{h} \rrbracket\right), \\
& \lambda_{h}=-\frac{\langle k\rangle}{2 \delta} \boldsymbol{n} \cdot \llbracket \boldsymbol{n} u_{h} \rrbracket+\langle k\rangle \boldsymbol{n} \cdot\left\{\nabla u_{h}\right\} .
\end{aligned}
$$

The test functions may be taken as

$$
\begin{aligned}
& \kappa_{h}=\frac{\left\{k v_{h}\right\}}{\{k\}}-\frac{\delta}{2\{k\}} \llbracket k \boldsymbol{n} \cdot \nabla v_{h} \rrbracket, \\
& \mu_{h}=-\frac{\langle k\rangle}{2 \delta} \boldsymbol{n} \cdot \llbracket \boldsymbol{n} v_{h} \rrbracket+\langle k\rangle \boldsymbol{n} \cdot\left\{\nabla v_{h}\right\},
\end{aligned}
$$

with $v_{h} \in V_{h}$.

For Neumann edges the corresponding expressions are:

$$
\begin{aligned}
\gamma_{h} & =u_{h}-\frac{\delta}{k}\left(k \boldsymbol{n} \cdot \nabla u_{h}-q\right), \\
\lambda_{h} & =q, \\
\kappa_{h} & =v_{h}-\delta \boldsymbol{n} \cdot \nabla v_{h}, \\
\mu_{h} & =0 .
\end{aligned}
$$

Remark 3 It is obvious that other choices of test functions are possible, particularly if no care is put on whether the resulting formulation is symmetric or not. The ones chosen here automatically will yield adjoint consistency of the method to be designed. $\square$ 
We have that

$$
\begin{aligned}
- & \sum_{K}\left\langle\lambda_{h}, v_{h}\right\rangle_{\partial K}+\sum_{K}\left\langle\kappa_{h}, \lambda_{h}\right\rangle_{\partial K}-\sum_{K}\left\langle\mu_{h}, u_{h}-\gamma_{h}\right\rangle_{\partial K} \\
= & -\sum_{E}\left\langle-\frac{\langle k\rangle}{2 \delta} \llbracket \boldsymbol{n} u_{h} \rrbracket+\langle k\rangle\left\{\nabla u_{h}\right\}, \llbracket \boldsymbol{n} v_{h} \rrbracket\right\rangle_{E} \\
& -\sum_{E}\left\langle-\frac{\langle k\rangle}{2 \delta} \llbracket \boldsymbol{n} v_{h} \rrbracket+\langle k\rangle\left\{\nabla v_{h}\right\}, \llbracket \boldsymbol{n} u_{h} \rrbracket\right\rangle_{E} \\
& -\left\langle v_{h}, q\right\rangle_{\Gamma_{N}}+\left\langle\kappa_{h}, q\right\rangle_{\Gamma_{N}} \\
= & \sum_{E}\langle k\rangle \delta\left\langle\frac{1}{\delta} \llbracket \boldsymbol{n} v_{h} \rrbracket-\left\{\nabla v_{h}\right\}, \frac{1}{\delta} \llbracket \boldsymbol{n} u_{h} \rrbracket-\left\{\nabla u_{h}\right\}\right\rangle_{E}-\sum_{E}\langle k\rangle \delta\left\langle\left\{\nabla v_{h}\right\},\left\{\nabla u_{h}\right\}\right\rangle_{E} \\
& -\left\langle v_{h}, q\right\rangle_{\Gamma_{N}}+\left\langle\kappa_{h}, q\right\rangle_{\Gamma_{N}}
\end{aligned}
$$

Using this in (13) we obtain the method we propose: find $u_{h} \in V_{h}$ such that

$$
\begin{array}{rl}
\sum_{K} & k\left\langle\nabla v_{h}, \nabla u_{h}\right\rangle_{K}+\sum_{K}\left\langle v_{h}, \boldsymbol{a} \cdot \nabla u_{h}\right\rangle_{K}+\sum_{K} s\left\langle v_{h}, u_{h}\right\rangle_{K} \\
& +\sum_{E}\langle k\rangle \delta\left\langle\frac{1}{\delta} \llbracket \boldsymbol{n} v_{h} \rrbracket-\left\{\nabla v_{h}\right\}, \frac{1}{\delta} \llbracket \boldsymbol{n} u_{h} \rrbracket-\left\{\nabla u_{h}\right\}\right\rangle_{E}-\sum_{E}\langle k\rangle \delta\left\langle\left\{\nabla v_{h}\right\},\left\{\nabla u_{h}\right\}\right\rangle_{E} \\
& =\sum_{K} L_{K}\left(v_{h}\right)+\left\langle v_{h}, q\right\rangle_{\Gamma_{N}},
\end{array}
$$

for all $v_{h} \in V_{h}$.

Remark 4 Let us make several remarks about the numerical approximation just obtained:

- Essentially, we have recovered the so called interior penalty method. Note that we may take $\delta=\delta_{0} h$, with $\delta_{0}<1 / 2$, which is now the algorithmic parameter of the formulation. This penalty term is not added "a posteriori", as usual, but comes from the reconstruction of the unknown in terms of its values in the element interiors and its traces on the element boundaries.

- Adjoint consistency is ensured. This is a result of taking the test functions as indicated in (23) and (24), and therefore it is quite natural when looking at the hybrid form of the problem.

- Several different methods may be devised within the framework presented. Among the possible alternatives there are different approximations of the diffusive fluxes using finite-difference-like expressions or other expressions of the test functions.

- The most salient feature of the method obtained with respect to the classical interior penalty method is the use of harmonic averages rather than arithmetic ones. This has been derived from the continuity of the fluxes, but has been used earlier for the convection-diffusion equation in [17], and later in [18], for example. It has also beed used in a domain decomposition method in [10]. 


\section{Stokes' problem}

In this section we apply the idea of Section 2 to the Stokes problem. Contrary to the previous section, we will skip most of the details and state only the results. The omitted steps are merely algebraic manipulations that are similar to those done for the convection-diffusion equation.

\subsection{Problem statement}

The Stokes problem consists of finding $\boldsymbol{u}: \Omega \longrightarrow \mathbb{R}^{d}$ and $p: \Omega \longrightarrow \mathbb{R}$ such that

$$
\begin{aligned}
-\nabla \cdot(\nu \nabla \boldsymbol{u})+\nabla p & =\boldsymbol{f} \\
\nabla \cdot \boldsymbol{u} & =0
\end{aligned}
$$

where $\nu>0$ is the viscosity, which we will consider constant within each element of the finite element approximation we will introduce, and $f$ is the vector of body forces. For simplicity, the boundary condition $\boldsymbol{u}=\mathbf{0}$ will be assumed and, as in the previous section, it will be applied in an essential manner. Note also that we have not used the symmetric gradient of $\boldsymbol{u}$ in the viscous term. This would complicate a little the approximation of the tractions, as we shall see, but could also be used without major difficulty.

When the problem is written in a hybrid form, which is a straightforward exercise, the identification with the abstract problem of Section 2 is obtained noting that the unknown $u$ and the fluxes $\lambda$ are

$$
\begin{aligned}
u & =[\boldsymbol{u}, p] \in \mathbb{R}^{d} \times \mathbb{R}, \\
\lambda & =\left[\boldsymbol{\lambda}_{u}, \lambda_{p}\right]=\left[-p \boldsymbol{n}+\nu \partial_{n} \boldsymbol{u}, 0\right] \in \mathbb{R}^{d} \times \mathbb{R} .
\end{aligned}
$$

\subsection{Discontinuous Galerkin approximation}

The dG finite element method to be proposed has again the structure (13), now with

$$
B([\boldsymbol{u}, p],[\boldsymbol{v}, q])=(\nu \nabla \boldsymbol{u}, \nabla \boldsymbol{v})-(p, \nabla \cdot \boldsymbol{v})+(q, \nabla \cdot \boldsymbol{u}) .
$$

Les us consider now a Galerkin finite element approximation of the hybrid problem, with the unknowns identified with a subscript $h$ as usual. If we impose the continuity of the discrete fluxes in a manner similar to that used for the convection-diffusion equation we will have that:

$$
\begin{aligned}
\mathbf{0} & =\boldsymbol{\lambda}_{u, h, 1}+\boldsymbol{\lambda}_{u, h, 2} \\
& =-p_{h, 1} \boldsymbol{n}_{1}-p_{h, 2} \boldsymbol{n}_{2}+\nu_{1} \boldsymbol{n}_{1} \cdot \nabla \boldsymbol{u}_{h, 1}+\nu_{2} \boldsymbol{n}_{2} \cdot \nabla \boldsymbol{u}_{h, 2} \\
& \approx-\llbracket p_{h} \boldsymbol{n} \rrbracket+\llbracket \nu \partial_{n} \boldsymbol{u}_{h} \rrbracket+\frac{1}{\delta}\left[\left(\nu_{1}+\nu_{2}\right) \gamma_{u, h}-\nu_{1} \boldsymbol{u}_{h, 1}-\nu_{2} \boldsymbol{u}_{h, 2}\right],
\end{aligned}
$$

where $\gamma_{u, h}$ are the traces of $\boldsymbol{u}$. These can be determined from this last expression, yielding:

$$
\gamma_{u, h}=\frac{\left\{\nu \boldsymbol{u}_{h}\right\}}{\{\nu\}}-\frac{\delta}{2\{\nu\}}\left(-\llbracket p_{h} \boldsymbol{n} \rrbracket+\llbracket \nu \partial_{n} \boldsymbol{u}_{h} \rrbracket\right) .
$$

This allows us to compute the fluxes, which after algebraic manipulations are:

$$
\boldsymbol{\lambda}_{u, h}=-\frac{\langle\nu\rangle}{2 \delta} \boldsymbol{n} \cdot \llbracket \boldsymbol{n} \otimes \boldsymbol{u}_{h} \rrbracket+\langle\nu\rangle \boldsymbol{n} \cdot\left\{\nabla \boldsymbol{u}_{h}\right\}-\boldsymbol{n}\langle\nu\rangle\left\{\frac{p_{h}}{\nu}\right\} .
$$


With these fluxes in terms of the unknowns we can already formulate the $\mathrm{dG}$ method for the Stokes problem we propose, which consists of finding $\left[\boldsymbol{u}_{h}, p_{h}\right]$ in the appropriate dG spaces $V_{h} \times Q_{h}$ such that

$$
\begin{aligned}
& \sum_{K}\left\langle\nu \nabla \boldsymbol{u}_{h}, \nabla \boldsymbol{v}_{h}\right\rangle_{K}-\sum_{K}\left\langle p_{h}, \nabla \cdot \boldsymbol{v}_{h}\right\rangle_{K}+\sum_{K}\left\langle q_{h}, \nabla \cdot \boldsymbol{u}_{h}\right\rangle_{K} \\
& \quad+\sum_{E}\langle\nu\rangle\left\langle-\left\{\nabla \boldsymbol{u}_{h}\right\}+\left\{\frac{p_{h}}{\nu}\right\} \boldsymbol{I}, \llbracket \boldsymbol{n} \otimes \boldsymbol{v}_{h} \rrbracket\right\rangle_{E}+\sum_{E}\langle\nu\rangle\left\langle-\left\{\nabla \boldsymbol{v}_{h}\right\}-\left\{\frac{q_{h}}{\nu}\right\} \boldsymbol{I}, \llbracket \boldsymbol{n} \otimes \boldsymbol{u}_{h} \rrbracket\right\rangle_{E} \\
& \quad+\sum_{E} \frac{\langle\nu\rangle}{2 \delta}\left\langle\llbracket \boldsymbol{n} \otimes \boldsymbol{u}_{h} \rrbracket, \llbracket \boldsymbol{n} \otimes \boldsymbol{v}_{h} \rrbracket\right\rangle_{E}=\sum_{K}\left\langle\boldsymbol{f}, \boldsymbol{v}_{h}\right\rangle_{K},
\end{aligned}
$$

for all test functions $\left[\boldsymbol{v}_{h}, q_{h}\right] \in V_{h} \times Q_{h}$.

Remark 5 Essentially, we have recovered the interior penalty method for the Stokes problem with two major differences with respect to what would be a standard approach:

1. The use of harmonic averages of the viscosity $\nu$ in the boundary terms.

2. The scaling of the pressure and the pressure test function by $\nu$ in the terms involving tractions on the element boundaries.

As for the convection-diffusion equation, adjoint consistency is ensured.

Note that the algebraic system resulting from this discrete variational problem may be rendered symmetric simply by changing the sign of $q_{h}$, as it is usually done for the conforming approximation to Stokes' problem.

\subsection{Stabilized finite element problem}

In the previous subsection we have considered the Galerkin finite element approximation to Stokes' problem. In order to be stable, appropriate choices for the velocity and pressure spaces, $V_{h}$ and $Q_{h}$ are required. Here we briefly present a stabilized finite element method which is aimed to allow for any interpolation spaces for velocity and pressure. Since it is neither our purpose to give a full heuristic motivation of the method, nor to analyze it, we will simply indicate how to motivate it. We refer to [26] for the analysis of different $\mathrm{dG}$ approximations of the Stokes problem.

Our way to design stabilized finite element methods is based on the so called Variational Multiscale (VMS) concept [22]. In the simplest case we will consider, we may split the unknown in the interior of the elements as $\boldsymbol{u} \approx \boldsymbol{u}_{h}+\boldsymbol{u}^{\prime}$, and leave the pressure as $p \approx p_{h}$. The field $\boldsymbol{u}^{\prime}$ is in fact what needs to be approximated. We refer to it as the velocity subgrid scale, and is assumed to be much smaller than $\boldsymbol{u}_{h}$, at least as $h \rightarrow 0$. Likewise, we approximate $\gamma_{u} \approx \gamma_{u, h}+\gamma_{u}^{\prime}, \gamma_{u}^{\prime}$ being the trace of $\boldsymbol{u}^{\prime}$.

Neglecting the derivatives of $\boldsymbol{u}^{\prime}$ on the element edges and considering $\left|\boldsymbol{u}^{\prime}\right| \ll\left|\boldsymbol{u}_{h}\right|$, we only need to deal with the terms:

$$
\begin{aligned}
& \sum_{K}\left\langle\nu \nabla\left(\boldsymbol{u}_{h}+\boldsymbol{u}^{\prime}\right), \nabla \boldsymbol{v}_{h}\right\rangle_{K}+\sum_{K}\left\langle q_{h}, \nabla \cdot\left(\boldsymbol{u}_{h}+\boldsymbol{u}^{\prime}\right)\right\rangle_{K} \\
& \quad=\sum_{K}\left\langle\nu \nabla \boldsymbol{u}_{h}, \nabla \boldsymbol{v}_{h}\right\rangle_{K}+\sum_{K}\left\langle q_{h}, \nabla \cdot \boldsymbol{u}_{h}\right\rangle_{K} \\
& \quad-\sum_{K}\left\langle\boldsymbol{u}^{\prime}, \nu \Delta \boldsymbol{v}_{h}+\nabla q_{h}\right\rangle_{K}+\sum_{K}\left\langle\gamma_{u}^{\prime}, \nu \partial_{n} \boldsymbol{v}_{h}+q_{h} \boldsymbol{n}\right\rangle_{\partial K},
\end{aligned}
$$

where we have used the fact that $\nu$ is constant within each element domain $K$. 
The next step is to model $\boldsymbol{u}^{\prime}$ in each element domain $K$ and $\gamma_{u}^{\prime}$ on each edge $E$. We will not give the details of how to do this modelling, and refer for example to $[14,16]$. In fact, the approximation for $\gamma_{u}^{\prime}$ follows from what we have presented in the previous subsection. Expression (27) should now be equal to $\gamma_{u, h}+\gamma_{u}^{\prime}$, understanding this equality as a splitting of the space of traces with one component in the trace finite element space and another in the trace subgrid scale space. Taking the effect of the projection as an algorithmic constant we model $\boldsymbol{u}^{\prime}$ and $\gamma_{u}^{\prime}$ as

$$
\begin{aligned}
\boldsymbol{u}^{\prime} & =\tau_{K}\left(\boldsymbol{f}+\nu \Delta \boldsymbol{u}_{h}-\nabla p_{h}\right), \quad \tau_{K}=c_{1} \frac{h^{2}}{\nu}, \\
\gamma_{u}^{\prime} & =\tau_{E}\left(-\llbracket p_{h} \boldsymbol{n} \rrbracket+\llbracket \nu \partial_{n} \boldsymbol{u}_{h} \rrbracket\right), \quad \tau_{E}=c_{2} h\left\langle\nu^{-1}\right\rangle
\end{aligned}
$$

where $c_{1}$ and $c_{2}$ are fixed algorithmic constants and $h$ is a characteristic size of either the element domain $K$ or of the edge $E$.

Inserting these expressions into (29) the stabilized finite element method we propose is: find $\left[\boldsymbol{u}_{h}, p_{h}\right] \in V_{h} \times Q_{h}$ such that

$$
\begin{aligned}
& \sum_{K}\left\langle\nu \nabla \boldsymbol{u}_{h}, \nabla \boldsymbol{v}_{h}\right\rangle_{K}-\sum_{K}\left\langle p_{h}, \nabla \cdot \boldsymbol{v}_{h}\right\rangle_{K}+\sum_{K}\left\langle q_{h}, \nabla \cdot \boldsymbol{u}_{h}\right\rangle_{K} \\
& \quad+\sum_{K} \tau_{K}\left\langle-\nu \Delta \boldsymbol{u}_{h}+\nabla p_{h}, \nu \Delta \boldsymbol{v}_{h}+\nabla q_{h}\right\rangle_{K}+\sum_{E} \tau_{E}\left\langle\llbracket-\nu \partial_{h} \boldsymbol{u}_{h}+p_{h} \boldsymbol{n} \rrbracket, \llbracket \nu \partial_{n} \boldsymbol{v}_{h}+q_{h} \boldsymbol{n} \rrbracket\right\rangle_{E} \\
& \quad+\sum_{E}\langle\nu\rangle\left\langle-\left\{\nabla \boldsymbol{u}_{h}\right\}+\left\{\frac{p_{h}}{\nu}\right\} \boldsymbol{I}, \llbracket \boldsymbol{n} \otimes \boldsymbol{v}_{h} \rrbracket\right\rangle_{E}+\sum_{E}\langle\nu\rangle\left\langle-\left\{\nabla \boldsymbol{v}_{h}\right\}-\left\{\frac{q_{h}}{\nu}\right\} \boldsymbol{I}, \llbracket \boldsymbol{n} \otimes \boldsymbol{u}_{h} \rrbracket\right\rangle_{E} \\
& \quad+\sum_{E} \frac{\langle\nu\rangle}{2 \delta}\left\langle\llbracket \boldsymbol{n} \otimes \boldsymbol{u}_{h} \rrbracket, \llbracket \boldsymbol{n} \otimes \boldsymbol{v}_{h} \rrbracket\right\rangle_{E}=\sum_{K}\left\langle\boldsymbol{f}, \boldsymbol{v}_{h}\right\rangle_{K}+\sum_{K} \tau_{K}\left\langle\boldsymbol{f}, \nu \Delta \boldsymbol{v}_{h}+\nabla q_{h}\right\rangle_{K},
\end{aligned}
$$

for all test functions $\left[\boldsymbol{v}_{h}, q_{h}\right] \in V_{h} \times Q_{h}$. The parameters $\tau_{K}$ and $\tau_{E}$ are given by (30) and (31), respectively.

Formulation (32) is the stabilized dG method that we propose. Even though it is not our intention here to do its numerical analysis, which will be presented elsewhere, it is able to yield stable solutions for any pair of velocity-pressure finite element spaces $V_{h}$ and $Q_{h}$. In particular, equal interpolation will be used in the numerical examples.

Remark 6 In [13] the authors propose another dG formulation of the Stokes problem that allows equal order interpolation; the formulation therein includes a term of the form $\sum_{E}\left\langle\llbracket p_{h} \boldsymbol{n} \rrbracket \text {, } \llbracket q_{h} \boldsymbol{n} \rrbracket\right\rangle_{E}$, and therefore it is only consistent for smooth enough pressures, namely $p \in H^{1}(\Omega)$. This method would not converge to solutions with minimum regularity. In our case, consistency is ensured by the inclusion of the whole traction $-\nu \partial_{n} \boldsymbol{u}_{h}+p_{h} \boldsymbol{n}$ in the jump.

\section{Darcy's problem}

Let us consider now Darcy's problem. We will treat it very briefly, since as we shall explain in this case the hybrid point of view does not introduce any modification to classical dG methods for this problem. 


\subsection{Problem statement}

The problem we consider now consists of finding $\boldsymbol{u}: \Omega \longrightarrow \mathbb{R}^{d}$ and $p: \Omega \longrightarrow \mathbb{R}$ such that

$$
\begin{aligned}
\sigma \boldsymbol{u}+\nabla p & =\boldsymbol{f} \\
\nabla \cdot \boldsymbol{u} & =g
\end{aligned}
$$

where $\sigma>0$ is the porosity coefficient, which we will consider constant within each element of the finite element approximation, $f$ is the vector of body forces and $g$ is a prescribed mass flux satisfying the compatibility condition $(g, 1)=0$ since, for simplicity, the boundary condition $\boldsymbol{n} \cdot \boldsymbol{u}=0$ on $\partial \Omega$ will be assumed. Once again, it will be applied in an essential manner.

The weak variational form of the problem admits two functional settings (see [6] for a discussion). Here we will concentrate in the so called $H(\operatorname{div}, \Omega) \times L^{2}(\Omega)$ (mixed dual) formulation, which in the irreducible case consists of finding $\boldsymbol{u} \in V=\{\boldsymbol{v} \in H(\operatorname{div}, \Omega) \mid \boldsymbol{n} \cdot \boldsymbol{v}=0$ on $\partial \Omega\}$ and $p \in Q=$ $L^{2}(\Omega) / \mathbb{R}$ such that

$$
B([\boldsymbol{u}, p],[\boldsymbol{v}, q]):=(\sigma \boldsymbol{u}, \boldsymbol{v})-(p, \nabla \cdot \boldsymbol{v})+(q, \nabla \cdot \boldsymbol{u})=\langle\boldsymbol{f}, \boldsymbol{v}\rangle+\langle g, q\rangle=: L([\boldsymbol{v}, q]),
$$

for all $v \in V, q \in Q$.

The continuous hybrid formulation has the structure described in Section 2, now with the unknown $u$ and the fluxes $\lambda$ given by

$$
\begin{aligned}
u & =[\boldsymbol{u}, p] \in \mathbb{R}^{d} \times \mathbb{R}, \\
\lambda & =\left[\boldsymbol{\lambda}_{u}, \lambda_{p}\right]=[-p \boldsymbol{n}, 0] \in \mathbb{R}^{d} \times \mathbb{R} .
\end{aligned}
$$

\subsection{Discontinuous Galerkin approximation}

The discrete hybrid finite element approximation to the problem consists of finding $\boldsymbol{u}_{h}, p_{h}, \boldsymbol{\lambda}_{u, h}$ and $\gamma_{u, h}$ in the appropriate finite element spaces such that

$$
\begin{aligned}
\sum_{K} & \sigma\left\langle\boldsymbol{u}_{h}, \boldsymbol{v}_{h}\right\rangle_{K}-\sum_{K}\left\langle p_{h}, \nabla \cdot \boldsymbol{v}_{h}\right\rangle_{K}+\sum_{K}\left\langle q_{h}, \nabla \cdot \boldsymbol{u}_{h}\right\rangle_{K} \\
& -\sum_{K}\left\langle\boldsymbol{\lambda}_{u, h}, \boldsymbol{v}_{h}\right\rangle_{\partial K}+\sum_{K}\left\langle\boldsymbol{\lambda}_{u, h}, \boldsymbol{\kappa}_{u, h}\right\rangle_{\partial K}+\sum_{K}\left\langle\boldsymbol{\mu}_{u, h}, \boldsymbol{u}_{h}-\gamma_{u, h}\right\rangle_{\partial K} \\
& =\sum_{K}\left\langle\boldsymbol{f}, \boldsymbol{v}_{h}\right\rangle_{K}+\sum_{K}\left\langle g, q_{h}\right\rangle_{K},
\end{aligned}
$$

for all test functions $\boldsymbol{v}_{h}, q_{h}, \boldsymbol{\mu}_{u, h}$ and $\boldsymbol{\kappa}_{u, h}$.

Contrary to the previous problems, the fluxes in (33) do not contain any derivative of the unknown. Moreover, they depend on $p$, whose trace does not appear in the hybrid formulation of the problem. However, it is particularly simple to design an expression for these fluxes that satisfies a priori the continuity imposed weakly in (34). The simplest way to achieve this is to model the fluxes and their test functions as

$$
\boldsymbol{\lambda}_{u, h}=-\left\{p_{h}\right\} \boldsymbol{n}, \quad \boldsymbol{\mu}_{u, h}=-\left\{q_{h}\right\} \boldsymbol{n},
$$

on each edge $E$. With these expressions inserted into (34) we recover the structure (13). The traces $\gamma_{u, h}$ and their test functions are not required (note that only the continuity of the normal component of $\boldsymbol{u}_{h}$ is weakly prescribed in (34) when (35) is used). It is observed that (35) coincides with (28) when the viscosity vanishes. In fact, we could have treated Brinkman's problem including the limiting cases of zero porosity (Stokes) and zero viscosity (Darcy), as in the unified framework proposed in [2]. 
Nevertheless, the formulation obtained with the procedure described is a standard dG approximation to Darcy's problem without stabilization terms, and thus in this case the hybrid point of view does not lead to any modification of standard methods.

We refer to [3] for dG finite element approximations to Darcy's problem including stabilization terms.

\section{Maxwell's problem}

\subsection{Problem statement}

The last problem we consider is Maxwell's problem, which consists of finding $\boldsymbol{u}: \Omega \longrightarrow \mathbb{R}^{d}$ and $p: \Omega \longrightarrow \mathbb{R}$ such that

$$
\begin{aligned}
\nabla \times(\nu \nabla \times \boldsymbol{u})-\varepsilon \nabla p & =\boldsymbol{f}, \\
-\nabla \cdot(\varepsilon \boldsymbol{u}) & =0,
\end{aligned}
$$

where $\nu>0$ and $\varepsilon>0$ are physical properties, again considered piecewise constant, and $\boldsymbol{f}$ is a forcing vector. The simplest boundary conditions $\boldsymbol{n} \times \boldsymbol{u}=\mathbf{0}$ and $p=0$ on $\partial \Omega$ will be adopted. Note that the solution of the continuous problem is $p=0$. This unknown is in fact an artifact to impose condition (37) at the discrete level. See $[5,6,4]$ and references therein for a discussion.

As for Darcy's problem, the weak variational form of the problem admits two functional settings (see [6]). Here we will consider only the $H(\operatorname{curl}, \Omega) \times H^{1}(\Omega)$ formulation, which allows one to capture singular solutions. It consists of finding $\boldsymbol{u} \in V=\{\boldsymbol{v} \in H(\operatorname{curl}, \Omega) \mid \boldsymbol{n} \times \boldsymbol{v}=\mathbf{0}$ on $\partial \Omega\}$ and $p \in Q=H_{0}^{1}(\Omega)$ such that

$$
B([\boldsymbol{u}, p],[\boldsymbol{v}, q]):=(\nu \nabla \times \boldsymbol{u}, \nabla \times \boldsymbol{v})-(\varepsilon \nabla p, \boldsymbol{v})+(\varepsilon \nabla q, \boldsymbol{u})=\langle\boldsymbol{f}, \boldsymbol{v}\rangle=: L([\boldsymbol{v}, q]),
$$

for all $\boldsymbol{v} \in V, q \in Q$.

Once again, the continuous hybrid formulation has the structure described in Section 2, now with the unknown $u$ and the fluxes $\lambda$ given by

$$
\begin{aligned}
u & =[\boldsymbol{u}, p] \in \mathbb{R}^{d} \times \mathbb{R}, \\
\lambda & =\left[\boldsymbol{\lambda}_{u}, \lambda_{p}\right]=[-\boldsymbol{n} \times(\nu \nabla \times \boldsymbol{u}), \boldsymbol{n} \cdot(\varepsilon \boldsymbol{u})] \in \mathbb{R}^{d} \times \mathbb{R} .
\end{aligned}
$$

\subsection{Discontinuous Galerkin approximation}

Let us explicitly write down the discrete hybrid finite element approximation to Maxwell's problem. Let $\left[\boldsymbol{u}_{h}, p_{h}\right]$ be the approximation sought to $[\boldsymbol{u}, p],\left[\boldsymbol{\lambda}_{u, h}, \lambda_{p, h}\right]$ the approximation to $\left[\boldsymbol{\lambda}_{u}, \lambda_{p}\right]$ and $\left[\boldsymbol{\gamma}_{u, h}, \gamma_{p, h}\right]$ the approximation to the traces of $[\boldsymbol{u}, p]$ on the edges of the finite element partition. These finite element unknowns are solution of the problem

$$
\begin{aligned}
& \sum_{K} B_{K}\left(\left[\boldsymbol{u}_{h}, p_{h}\right],\left[\boldsymbol{v}_{h}, q_{h}\right]\right)-\sum_{K}\left\langle\boldsymbol{\lambda}_{u, h}, \boldsymbol{v}_{h}\right\rangle_{\partial K}-\sum_{K}\left\langle\lambda_{p, h}, q_{h}\right\rangle_{\partial K}=\sum_{K} L_{K}\left(\left[\boldsymbol{v}_{h}, q_{h}\right]\right), \\
& \sum_{K}\left\langle\boldsymbol{\kappa}_{u, h}, \boldsymbol{\lambda}_{u, h}\right\rangle_{\partial K}+\sum_{K}\left\langle\kappa_{p, h}, \lambda_{p, h}\right\rangle_{\partial K}=0, \\
& \sum_{K}\left\langle\boldsymbol{\mu}_{u, h}, \boldsymbol{u}_{h}-\gamma_{u, h}\right\rangle_{\partial K}+\sum_{K}\left\langle\mu_{p, h}, p_{h}-\gamma_{p, h}\right\rangle_{\partial K}=0,
\end{aligned}
$$

which has to hold for all test functions $\left[\boldsymbol{v}_{h}, q_{h}\right],\left[\boldsymbol{\mu}_{u, h}, \mu_{p, h}\right]$ and $\left[\boldsymbol{\kappa}_{u, h}, \kappa_{p, h}\right]$ in the corresponding finite element spaces. 
To proceed in this case as explained in Section 2, we need to calculate the fluxes in terms of the traces and determine the latter by imposing continuity of the former. Now this idea has to be applied componentwise, since the fluxes have the two components $\left[\boldsymbol{\lambda}_{u, h}, \lambda_{p, h}\right]$. Imposing continuity of the first component, that is to say, that $\llbracket \boldsymbol{n} \times \nu \nabla \times \boldsymbol{u} \rrbracket=\mathbf{0}$, and using a similar procedure to approximate derivatives as in Sections 3 and 4, we get

$$
\begin{aligned}
& \boldsymbol{\gamma}_{u, h}=\frac{\left\{\nu \boldsymbol{u}_{h}\right\}}{\{\nu\}}+\frac{\delta}{2\{\nu\}} \llbracket \boldsymbol{n} \times \nu \nabla \times \boldsymbol{u}_{h} \rrbracket, \\
& \boldsymbol{\lambda}_{u, h}=\frac{\langle\nu\rangle}{\delta} \boldsymbol{n} \times\left\{\boldsymbol{n} \times \boldsymbol{u}_{h}\right\}-\langle\nu\rangle \boldsymbol{n} \times\left\{\nabla \times \boldsymbol{u}_{h}\right\} .
\end{aligned}
$$

Continuity of the second component of the fluxes follows from $\llbracket \boldsymbol{n} \cdot(\varepsilon \boldsymbol{u}) \rrbracket=0$. This condition does not involve any derivative of the unknown. The simplest way to fulfil it is to take

$$
\lambda_{p, h}=\boldsymbol{n} \cdot\left\{\varepsilon \boldsymbol{u}_{h}\right\} .
$$

With the choices (43) and (44) the fluxes are automatically continuous across interelement boundaries and, as explained earlier, traces are not required in the discrete hybrid finite element problem. In particular, the boundary terms in (39)-(41) are

$$
\begin{aligned}
- & \sum_{K}\left\langle\boldsymbol{\lambda}_{u, h}, \boldsymbol{v}_{h}\right\rangle_{\partial K}-\sum_{K}\left\langle\lambda_{p, h}, q_{h}\right\rangle_{\partial K}+\sum_{K}\left\langle\boldsymbol{\mu}_{u, h}, \boldsymbol{u}_{h}\right\rangle_{\partial K}+\sum_{K}\left\langle\mu_{p, h}, p_{h}\right\rangle_{\partial K} \\
= & \sum_{E} \frac{4\langle\nu\rangle}{\delta}\left\langle\left\{\boldsymbol{n} \times \boldsymbol{u}_{h}\right\},\left\{\boldsymbol{n} \times \boldsymbol{v}_{h}\right\}\right\rangle_{E} \\
& -\sum_{E} 2\langle\nu\rangle\left\langle\nabla \times \boldsymbol{u}_{h},\left\{\boldsymbol{n} \times \boldsymbol{v}_{h}\right\}\right\rangle_{E}-\sum_{E} 2\langle\nu\rangle\left\langle\nabla \times \boldsymbol{v}_{h},\left\{\boldsymbol{n} \times \boldsymbol{u}_{h}\right\}\right\rangle_{E} \\
& -\sum_{E}\left\langle\left\{\varepsilon \boldsymbol{u}_{h}\right\}, \llbracket \boldsymbol{n} q_{h} \rrbracket\right\rangle_{E}+\sum_{E}\left\langle\left\{\varepsilon \boldsymbol{v}_{h}\right\}, \llbracket \boldsymbol{n} p_{h} \rrbracket\right\rangle_{E},
\end{aligned}
$$

and therefore the $\mathrm{dG}$ method for the Maxwell problem we propose consists of finding $\left[\boldsymbol{u}_{h}, p_{h}\right]$ in the appropriate $\mathrm{dG}$ spaces $V_{h} \times Q_{h}$ such that

$$
\begin{aligned}
& \sum_{K} \nu\left\langle\nabla \times \boldsymbol{u}_{h}, \nabla \times \boldsymbol{v}_{h}\right\rangle_{K}-\sum_{K} \varepsilon\left\langle\nabla p_{h}, \boldsymbol{v}_{h}\right\rangle_{K}+\sum_{K} \varepsilon\left\langle\nabla q_{h}, \boldsymbol{u}_{h}\right\rangle_{K} \\
& \quad+\sum_{E} \frac{4\langle\nu\rangle}{\delta}\left\langle\left\{\boldsymbol{n} \times \boldsymbol{u}_{h}\right\},\left\{\boldsymbol{n} \times \boldsymbol{v}_{h}\right\}\right\rangle_{E} \\
& \quad-\sum_{E} 2\langle\nu\rangle\left\langle\nabla \times \boldsymbol{u}_{h},\left\{\boldsymbol{n} \times \boldsymbol{v}_{h}\right\}\right\rangle_{E}-\sum_{E} 2\langle\nu\rangle\left\langle\nabla \times \boldsymbol{v}_{h},\left\{\boldsymbol{n} \times \boldsymbol{u}_{h}\right\}\right\rangle_{E} \\
& \quad-\sum_{E}\left\langle\left\{\varepsilon \boldsymbol{u}_{h}\right\}, \llbracket \boldsymbol{n} q_{h} \rrbracket\right\rangle_{E}+\sum_{E}\left\langle\left\{\varepsilon \boldsymbol{v}_{h}\right\}, \llbracket \boldsymbol{n} p_{h} \rrbracket\right\rangle_{E}=\sum_{K}\left\langle\boldsymbol{f}, \boldsymbol{v}_{h}\right\rangle_{K},
\end{aligned}
$$

for all test functions $\left[\boldsymbol{v}_{h}, q_{h}\right] \in V_{h} \times Q_{h}$. Alternative $\mathrm{dG}$ formulations for the Maxwell problem can be found e.g. in $[21,20,19,24]$. The main novelty of our approach is the introduction of the harmonic averages.

\subsection{Stabilized finite element problem}

Formulation (45) is of Galerkin type. To ensure stability, appropriate interpolation spaces for $\boldsymbol{u}_{h}$ and $p_{h}$ need to be chosen. In order to avoid any restriction in the choice of these interpolating spaces, a stabilized finite element formulation combining continuous and discontinuous interpolations was proposed 
in [4]. In the particular case of elementwise discontinuous approximations, the method proposed in the paper mentioned is applicable in the present context.

We do not intend to describe in detail the stabilization method we propose, but just to explain the essential idea. Let $\ell$ be a characteristic length of the computational domain $\Omega$. Since the solution to (36)-(37) is $p=0$, the solution $\boldsymbol{u}$ remains unaltered if the Laplacian-like term $-\ell^{2} \nabla \cdot(\varepsilon \nabla p)$ is added to the left-hand-side of (37). In the discrete problem, this allows one to get $H^{1}$-control on $p_{h}$. Note that the length scale $\ell$ is needed to ensure an adequate scalability of the problem.

To ensure stability of $\boldsymbol{u}_{h}$ in $H(\operatorname{curl}, \Omega)$ one needs to control $\nabla \times \boldsymbol{u}_{h}$ and $\boldsymbol{u}_{h}$ itself in $L^{2}(\Omega)$. The first control is straightforward from (45). It has been shown in [5] that the latter can be obtained if one is able to bound $h \nabla \cdot \boldsymbol{u}_{h}$, properly scaled, in $L^{2}(\Omega)$. This leads to the introduction of a least squares form of this term in (45).

With the two ideas described in mind, the modification of (45) that we propose consists of finding $\left[\boldsymbol{u}_{h}, p_{h}\right]$ in the appropriate dG spaces $V_{h} \times Q_{h}$ such that

$$
\begin{array}{|l}
\sum_{K} \nu\left\langle\nabla \times \boldsymbol{u}_{h}, \nabla \times \boldsymbol{v}_{h}\right\rangle_{K}-\sum_{K} \varepsilon\left\langle\nabla p_{h}, \boldsymbol{v}_{h}\right\rangle_{K}+\sum_{K} \varepsilon\left\langle\nabla q_{h}, \boldsymbol{u}_{h}\right\rangle_{K} \\
\quad+\sum_{K} \tau_{K}\left\langle\nabla \cdot\left(\varepsilon \boldsymbol{u}_{h}\right), \nabla \cdot\left(\varepsilon \boldsymbol{v}_{h}\right)\right\rangle_{K}+\sum_{E} \tau_{E}\left\langle\llbracket \varepsilon \boldsymbol{n} \cdot \boldsymbol{u}_{h} \rrbracket, \llbracket \varepsilon \boldsymbol{n} \cdot \boldsymbol{v}_{h} \rrbracket\right\rangle_{E} \\
\quad+\sum_{E} \frac{4\langle\nu\rangle}{\delta}\left\langle\left\{\boldsymbol{n} \times \boldsymbol{u}_{h}\right\},\left\{\boldsymbol{n} \times \boldsymbol{v}_{h}\right\}\right\rangle_{E} \\
\quad-\sum_{E} 2\langle\nu\rangle\left\langle\nabla \times \boldsymbol{u}_{h},\left\{\boldsymbol{n} \times \boldsymbol{v}_{h}\right\}\right\rangle_{E}-\sum_{E} 2\langle\nu\rangle\left\langle\nabla \times \boldsymbol{v}_{h},\left\{\boldsymbol{n} \times \boldsymbol{u}_{h}\right\}\right\rangle_{E} \\
\quad-\sum_{E}\left\langle\left\{\varepsilon \boldsymbol{u}_{h}\right\}, \llbracket \boldsymbol{n} q_{h} \rrbracket\right\rangle_{E}+\sum_{E}\left\langle\left\{\varepsilon \boldsymbol{v}_{h}\right\}, \llbracket \boldsymbol{n} p_{h} \rrbracket\right\rangle_{E} \\
+\sum_{K} \ell^{2} \varepsilon\left\langle\nabla p_{h}, \nabla q_{h}\right\rangle_{K}+\sum_{E} \ell^{2} \frac{\langle\varepsilon\rangle}{\delta}\left\langle\llbracket \boldsymbol{n} p_{h} \rrbracket, \llbracket \boldsymbol{n} q_{h} \rrbracket\right\rangle_{E}=\sum_{K}\left\langle\boldsymbol{f}, \boldsymbol{v}_{h}\right\rangle_{K},
\end{array}
$$

for all test functions $\left[\boldsymbol{v}_{h}, q_{h}\right] \in V_{h} \times Q_{h}$, and where the parameters $\tau_{K}$ and $\tau_{E}$ are given by

$$
\tau_{K}=c_{1} \frac{h^{2}}{\ell^{2} \varepsilon}, \quad \tau_{E}=c_{2} \frac{h\left\langle\varepsilon^{-1}\right\rangle}{\ell^{2}}
$$

$c_{1}$ and $c_{2}$ being algorithmic constants.

\section{Numerical examples}

Rather than proposing new methods, the aim of this paper has been to describe a framework to design discontinuous Galerkin methods from hybrid formulations. However, in doing so we have been led to the use of harmonic averages rather than arithmetic averages of the physical properties when computing certain terms on the element edges. The aim of this section is to compare both possibilities for two problems in which, to our knowledge, harmonic averages have not been used before, namely, the Stokes problem and the Maxwell problem. Therefore, we will compare the formulations we have proposed with those obtained replacing $\langle\nu\rangle$ and $\left\langle\nu^{-1}\right\rangle$ by $\{\nu\}$ and $\left\{\nu^{-1}\right\}$, respectively.

For the numerical examples we have considered, the difference in the solution using the two approaches turn out to be very small. However, we observed an enormous difference in the performance of domain decomposition preconditioners for iterative solvers, when strong physical parameter jumps are 
present on the sub-domain interfaces. This is what we report next. We show that the use of harmonic averages leads to a much better performance of iterative solvers, with the number of iterations quite insensitive to the ratio of physical properties between neighboring elements. We start with Maxwell's problem and then show an example of Stokes' problem, since the former happens to be somewhat simpler than the latter. Triangular linear finite elements have been used for the discretization and the sub-domain linear systems have been solved with a multifrontal sparse direct solver.

\subsection{Maxwell's problem}

We consider in this example Maxwell's problem for two different materials, the first one located in $\Omega_{1}=[-1,0] \times[-1,1]$ and the second one in $\Omega_{2}=[0,1] \times[-1,1]$. The physical domain in which the test problem is solved is $\Omega \equiv \Omega_{1} \cup \Omega_{2}$. As indicated above, we compare the results obtained with the formulation proposed in this work, which uses harmonic averages of the physical coefficients in the $\mathrm{dG}$ terms, with a formulation that uses arithmetic averages.

Let us consider the test problem with exact solution

$$
\boldsymbol{u}(x, y)= \begin{cases}\left(x+y-x^{2}+x y+y^{2}+x^{3}-3 x^{2} y-3 x y^{2}+y^{3},\right. & \\ \left.2 x-y+\frac{1}{2} x^{2}+2 x y-\frac{1}{2} y^{2}-x^{3}-3 x^{2} y+3 x y^{2}+y^{3}\right) & \text { on } \Omega_{1} \\ \left(x+\bar{\varepsilon} y-\bar{\varepsilon} x^{2}+x y+\bar{\varepsilon} y^{2}+x^{3}-3 \bar{\varepsilon} x^{2} y-3 x y^{2}+\bar{\varepsilon} y^{3},\right. & \\ \left.(\bar{\varepsilon}+\bar{\nu}) x-y+\frac{1}{2} x^{2}+2 \bar{\varepsilon} x y-\frac{1}{2} y^{2}-\bar{\varepsilon} x^{3}-3 x^{2} y+3 \bar{\varepsilon} x y^{2}+y^{3}\right) & \text { on } \Omega_{2},\end{cases}
$$

where $\bar{\varepsilon}=\frac{\varepsilon_{1}}{\varepsilon_{2}}$ and $\bar{\nu}=\frac{\nu_{1}}{\nu_{2}}, \varepsilon_{i}$ and $\nu_{i}$ being the physical properties in $\Omega_{i}, i=1,2$. This function is the solution of problem (36)-(37) with the appropriate Dirichlet boundary conditions and zero forcing term.

The accuracy and convergence rates obtained for the two formulations considered (with harmonic means and with arithmetic means) are almost identical, and so, this is not a point that would justify one choice over the other one. The importance of using harmonic averages comes when considering domain decomposition techniques with material interfaces. In Figure 4 we show how the jump on the physical coefficient $\nu$ between the two materials deteriorates the estimated condition number of the final linear system. We have considered $\nu_{1}=1$ and $\nu_{2}=1,10,100,1000,10000$. Jumps on $\varepsilon$ do not alter the condition number substantially. The results have been presented for two mesh sizes, namely, $h=2^{-i}$, with $i=3,4$. As expected, the condition number deteriorates as $h \searrow 0$.

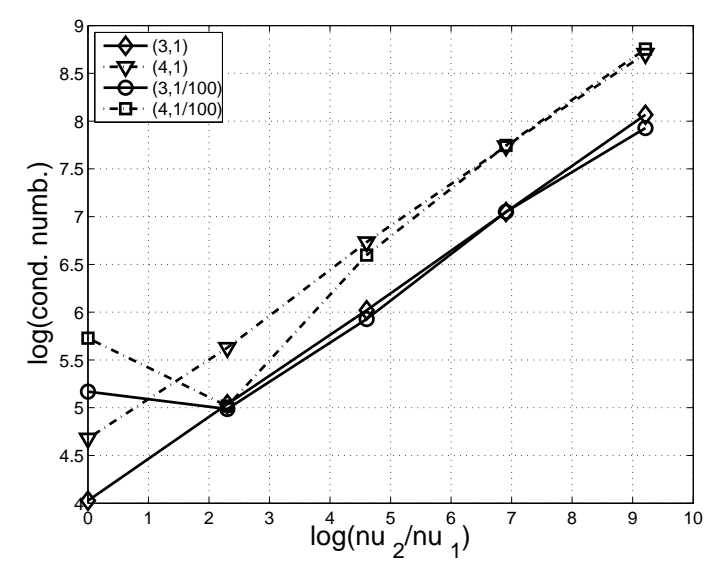

Figure 4: Condition number of the system matrix. The legend represents $\left(i, \varepsilon_{1} / \varepsilon_{2}\right)$ where $h=2^{-i}$.

As a result, the numerical approximation of electromagnetic problems that involve materials with very different physical properties becomes a challenging task due to the problems encountered when 
solving the linear system. Direct solvers will drastically reduce their accuracy and iterative methods their convergence. Let us write the resulting system matrix for the problem at hand as

$$
A=\left(\begin{array}{ll}
A_{11} & A_{12} \\
A_{21} & A_{22}
\end{array}\right),
$$

where the nodes in the set 1 are those that belong to finite elements $K \subset \Omega_{1}$, and analogously for the set 2. An important fact of the harmonic average is that, as $\nu_{2} \rightarrow \infty$ for $\nu_{1}$ fixed, the columns associated to 1-nodes remain bounded. Now, we can consider a block-Jacobi preconditioner based on a nonoverlapping mesh partition (see [27]), i.e. the matrix

$$
P=\left(\begin{array}{cc}
A_{11} & 0 \\
0 & A_{22}
\end{array}\right) .
$$

Using Richardson iterations with this preconditioner leads to block-Jacobi iterations. In Figure 5 we plot the number of Richardson iterations for the preconditioned system for the two different formulations against the value $\nu_{2}$, for $\nu_{1}=1$ fixed. We have considered different values for the mesh size $h$ and $\varepsilon$. We can observe from the results in this figure that the preconditioned matrix $P^{-1} A$ is much better conditioned for the harmonic averages, being insensitive to coefficient jumps. On the contrary, the preconditioned matrix is still ill-conditioned when using arithmetic averages as $\nu$ increases, and for $\nu$ large enough it does not converge at all, reaching the maximum of 5000 iterations imposed in the code.

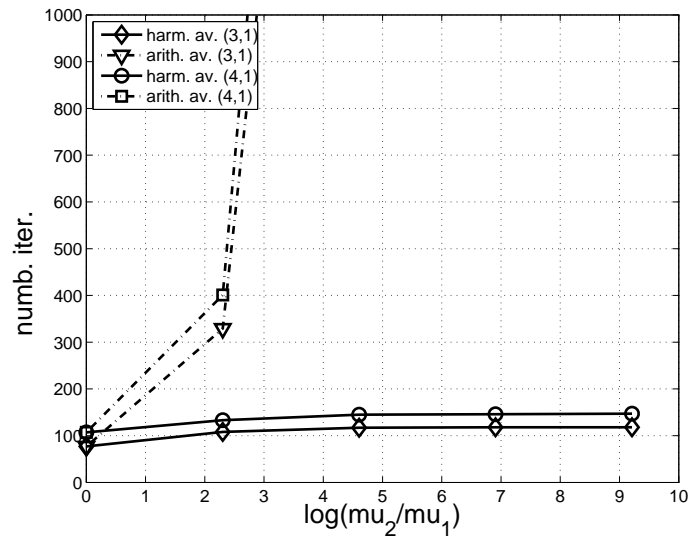

(a)

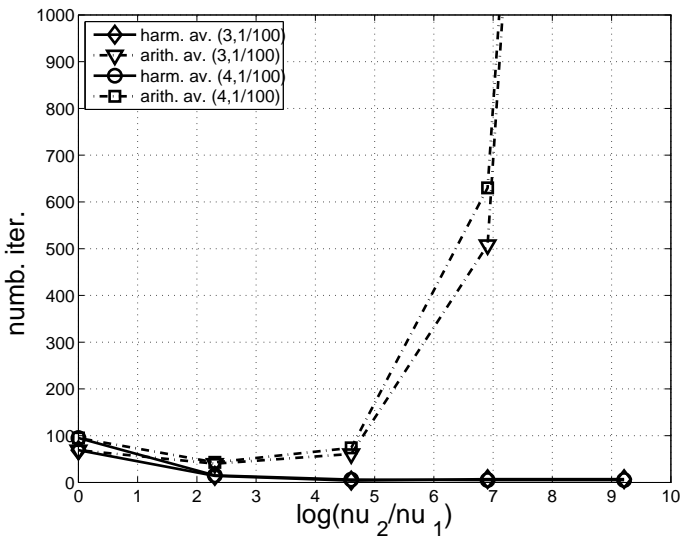

(b)

Figure 5: Number of Richardson iterations required till convergence, for the two different formulations: harmonic and arithmetic averages for $\nu$. The legend represents $\left(i, \varepsilon_{1} / \varepsilon_{2}\right)$ where $h=2^{-i}$.

\subsection{Stokes' problem}

Let us consider the numerical approximation of system (25)-(26) with a discontinuous value of $\nu$. This type of problem is usually found in the simulation of incompressible elastic materials. We consider the test problem with exact solution $\boldsymbol{u}=(x,-y)$ on $\Omega$ and $p=0$ on $\Omega_{1}$ and $p=\nu_{2}-\nu_{1}$ on $\Omega_{2}$. The resulting problem is solved by the GMRES iterative solver (see [25]) and the same preconditioner as 
above. This problem turns out to be more difficult to solve than the previous one, and non-orthonormal Richardson iterations failed to converge in all cases when using arithmetic averages.

In Figure 6(a) we show the number of iterations for $\nu_{1}=1$ and $\nu_{2}=1,10,100,1000,10000$. Again, two different values of $h=2^{-i}$, with $i=3,4$, have been considered. The harmonic averages lead to a much lower number of iterations, since it even improves as coefficient jumps increase. On the other hand, arithmetic averages lead to a harder to solve interface problem that requires a too large number of GMRES iterations. Estimates for the condition number of the preconditioned matrix are plotted in Figure 6(b). As expected, the condition number for harmonic averages remains bounded as $\frac{\nu_{1}}{\nu_{2}} \searrow 0$ whereas it explodes for arithmetic averages.

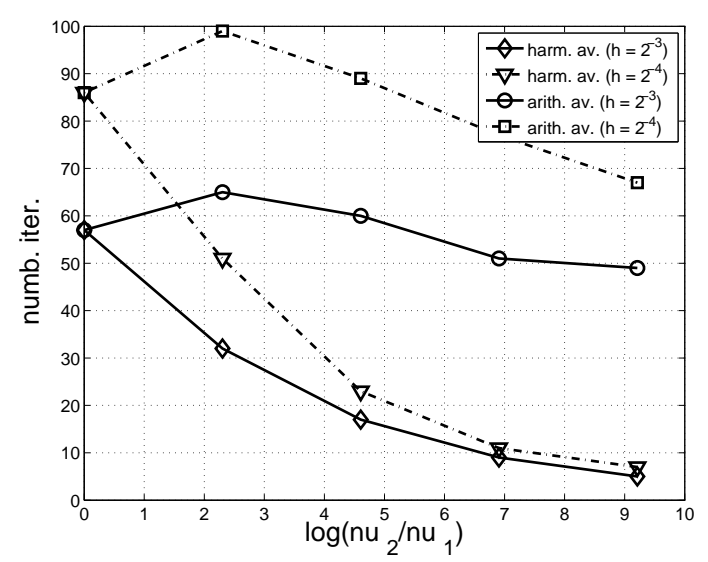

(a) Number of GMRES iterations

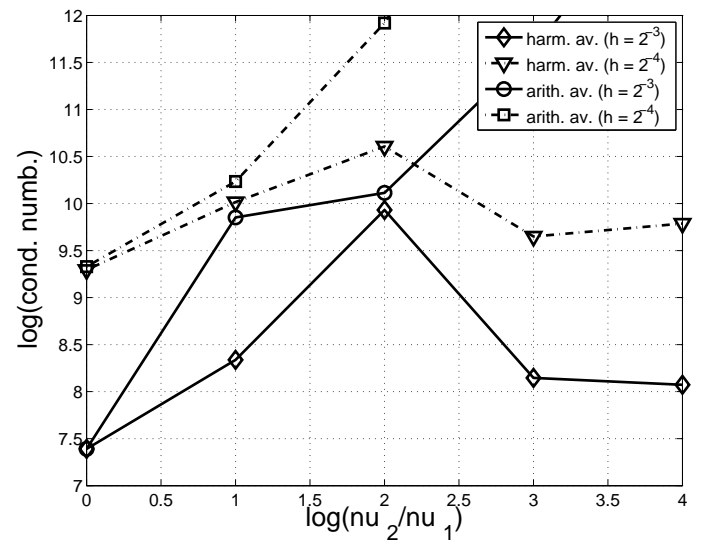

(b) Condition number

Figure 6: Number of GMRES iterations and condition number of the preconditioned matrix for harmonic and arithmetic averages and different values of $h$.

\section{Conclusions}

We have proposed a framework for the design of discontinuous Galerkin methods for elliptic problems based on hybrid three-field formulations. Starting with a problem posed in terms of the primal field, its fluxes and its traces on the element boundaries, the final problem is obtained after approximating the last two unknowns in terms of the first. In particular, fluxes are computed from a reconstruction of the unknown from the approximated primal variable and its traces, and are imposed to be continuous, an essential feature of the procedure we propose.

The general idea described has been applied to four different elliptic problems. The resulting methods are very similar to standard dG formulations, with some particular features. First, symmetry of the formulation, and the subsequent adjoint consistency, is shown to be a consequence of a natural choice for the test functions. Likewise, the stabilization term in interior penalty methods is shown to be related to the calculation of derivatives of the reconstructed unknown. It is also shown that in order to have continuity of fluxes harmonic averages rather than arithmetic averages of the physical properties need to be taken on the element edges. For the Stokes problem we have introduced an original scaling of the pressure that has been also motivated from the continuity of fluxes. Finally, we have described how to treat stabilization terms introduced in order to avoid restrictions in the choice of the interpolating spaces 
posed by inf-sup conditions.

Even if the main objective of this work has been to present a methodology to design dG methods rather than new formulations, we have also tested numerically the use of harmonic averages of physical variables on the element edges. For the Stokes and the Maxwell problem we are not aware of other methods that use this treatment of the physical coefficients. We have observed that the accuracy of the solution is similar to that obtained with arithmetic averages, but the performance of domain decomposition preconditioned iterative procedures with strong physical coefficient jumps is greatly improved.

\section{References}

[1] D.N. Arnold, F. Brezzi, B. Cockburn, and L.D. Marini. Unified analysis of discontinuous Galerkin methods for elliptic problems. SIAM Journal on Numerical Analysis, 39:1749-1779, 2002.

[2] S. Badia and R. Codina. Unified stabilized finite element formulations for the Stokes and the Darcy problems. SIAM Journal on Numerical Analysis, 47:1971-2000, 2009.

[3] S. Badia and R. Codina. Stabilized continuous and discontinuous Galerkin techniques for Darcy flow. Computer Methods in Applied Mechanics and Engineering, 199:1654-1667, 2010.

[4] S. Badia and R. Codina. A combined nodal continuous-discontinuous finite element formulation for the Maxwell problem. Applied Mathematics and Computation, 218:4276-4294, 2011.

[5] S. Badia and R. Codina. A nodal-based finite element approximation of the Maxwell problem suitable for singular solutions. SIAM Journal on Numerical Analysis, 50:398-417, 2012.

[6] S. Badia and R. Codina. Stokes, Maxwell and Darcy: a single finite element approximation for three model problems. Applied Numerical Mathematics, 62:246-263, 2012.

[7] F. Brezzi, B. Cockburn, L.D. Marini, and E. Süli. Stabilization mechanisms in discontinuous Galerkin finite element methods. Computer Methods in Applied Mechanics and Engineering, 195:3293-3310, 2006.

[8] F. Brezzi and D. Marini. Macro hybrid elements and domain decomposition methods. In Optimisation et controle, Actes du colloque organisé en l'honneur du soixantième anniversaire de Jean Céa, France, Avril 8-10 Toulouse, pages 89-96. Cepadues Editions, 1993.

[9] F. Brezzi and D. Marini. A threed-field domain decomposition method. Comtemporary Mathematics, 157:27-34, 1994.

[10] E. Burman and P. Zunino. A domain decomposition method based on weighted interior penalties for advection-diffusion-reaction problems. SIAM Journal on Numerical Analysis, 44:1612-1638, 2006.

[11] B. Cockburn, B. Dong, J. Guzman, M. Restelli, and R. Sacco. A hybridizable discontinuous Galerkin method for steady-state convection-diffusion-reaction problems. SIAM Journal on Scientific Computing, 31:3827-3846, 2009.

[12] B. Cockburn, J. Gopalakrishnan, and R. Lazarov. Unified hybridization of discontinuous Galerkin, mixed, and continuous Galerkin methods for second order elliptic problems. SIAM Journal on Numerical Analysis, 47:1319-1365, 2009. 
[13] B. Cockburn, G. Kanschat, and D. Schötzau. An equal-order DG method for the incompressible Navier-Stokes equations. Journal of Scientific Computing, 40:188-210, 2009. 10.1007/s10915008-9261-1.

[14] R. Codina. Analysis of a stabilized finite element approximation of the Oseen equations using orthogonal subscales. Applied Numerical Mathematics, 58:264-283, 2008.

[15] R. Codina and J. Baiges. Finite element approximation of transmission conditions in fluids and solids introducing boundary subgrid scales. International Journal for Numerical Methods in Engineering, 87:386-411, 2011.

[16] R. Codina, J. Principe, and J. Baiges. Subscales on the element boundaries in the variational two-scale finite element method. Computer Methods in Applied Mechanics and Engineering, 198:838-852, 2009.

[17] M. Dryja. On discontinuous Galerkin method for elliptic problems with discontinuous coefficients. Computational Methods in Applied Mathematics, 1:76-85, 2003.

[18] A. Ern, A. Stephansen, and P. Zunino. A discontinuous Galerkin method with weighted averages for advection-diffusion equations with locally vanishing and anisotropic diffusivity. IMA Journal of Numerical Analysis, 29:235-256, 2009.

[19] M. Grote, A. Schneebeli, and D. Schötzau. Interior penalty discontinuous Galerkin method for Maxwell's equations: energy norm error estimates. Journal of Computational and Applied Mathematics, 204(375-386), 2007.

[20] M.J. Grote, A. Schneebeli, and D. Schötzau. Interior penalty discontinuous Galerkin method for Maxwell's equations: optimal $L^{2}$-norm error estimates. IMA Journal of Numerical Analysis, 28:440-468, 2008.

[21] P. Houston, I. Perugia, and D. Schötzau. Mixed discontinuous Galerkin approximation of the Maxwell operator. SIAM Journal on Numerical Analysis, 42:434-459, 2004.

[22] T.J.R. Hughes, G.R. Feijóo, L. Mazzei, and J.B. Quincy. The variational multiscale method-a paradigm for computational mechanics. Computer Methods in Applied Mechanics and Engineering, 166:3-24, 1998.

[23] N.C. Nguyen, J. Peraire, and B. Cockburn. An implicit high-order hybridizable discontinuous Galerkin method for linear convection-diffusion equations. Journal of Computational Physics, 228:3232-3254, 2009.

[24] I. Perugia, D. Schötzau, and P. Monk. Stabilized interior penalty methods for the time-harmonic Maxwell equations. Computer Methods in Applied Mechanics and Engineering, 191:4675-4697, 2002.

[25] Y. Saad. Iterative methods for sparse linear systems. PWS Publishing, Boston, MA, 1996.

[26] D. Schötzau, Ch. Schwab, and A. Toselli. Mixed hp-DGFEM for incompressible flows. SIAM Journal on Numerical Analysis, 40(6):2171-2194, 2002.

[27] A. Toselli and O. Widlund. Domain decomposition methods-Algorithms and theory. Springer, 2005. 